
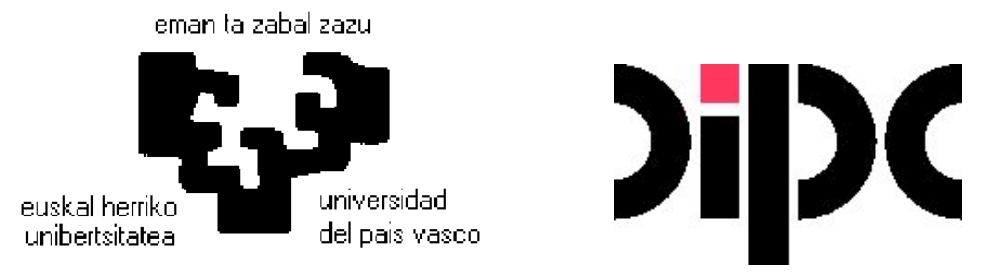

\title{
Heterogeneity of the segmental dynamics in lamellar phases of diblock copolymers
}

Master Thesis submitted in accordance with the requirements of the University of the Basque Country for the Master's degree in Nanoscience

by

\section{Mohammed Zakaria Slimani}

Thesis Director:

Dr. Ángel José Moreno Segurado

Donostia-San Sebastián, April 2011 



\section{Abstract}

By means of computer simulations, we investigate the segmental dynamics in the lamellar phase of a simple bead-spring model of diblock copolymers. We characterize the dynamic heterogeneity in the mean squared displacements and bond reorientations. This characterization is made as a function of both the position of the monomers along the chain and the distance to the nearest interface between consecutive domains. Both characterizations of the dynamic heterogeneity reveal moderate gradients of mobility in the investigated temperature range, which qualitatively probes relaxation time scales of up to hundreds nanoseconds. Namely, the obtained distribution of relaxation times spreads over about one decade. However, the extrapolation of the former analysis to lower temperatures leads to an increasing spread over several time decades. The spread mostly arises from monomers located at the inmediate neighborhood of the interface. Beyond such distances the structural relaxation approaches that of the homopolymer. Thus, the observed dynamic heterogeneity is esentially an interfacial effect. It does not originate from gradients of density over the domains. Indeed such gradients are absent and the local density within the domains is identical to that of the corresponding homopolymers. 


\section{Contents}

1 Introduction $\quad 5$

1.1 Copolymers ........................... 5

1.2 Dynamics in block copolymers . . . . . . . . . . . . . 9

1.3 Motivations of this work . . . . . . . . . . . 10

2 Simulations $\quad 11$

2.1 Background ............................ 11

2.2 Coarse-grained simulations of polymer systems . . . . . . . . . 12

2.3 Model and simulation details . . . . . . . . . . . . . . . 14

$\begin{array}{llr}3 & \text { Results } & 19\end{array}$

3.1 Static properties . . . . . . . . . . . . . . 19

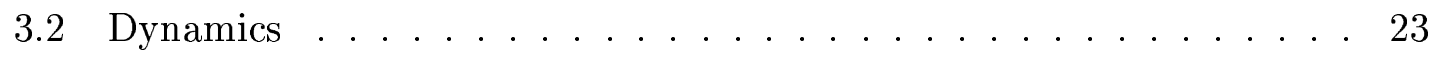

4 Discussion $\quad 33$

5 Conclusions $\quad 39$

$\begin{array}{ll}\text { Bibliography } & 40\end{array}$ 


\section{Chapter 1}

\section{Introduction}

\subsection{Copolymers}

Copolymers are polymer systems where the chains contain two or more different monomeric units. Let us start from two homopolymers which are thermodynamically uncompatible. Blending of both systems will lead to inmiscibility and therefore macrophase separation in a certain range of composition and temperature. On the contrary, copolymerization creates permanent chemical bonds between distinct monomeric units, which prevent macrophase separation. In this situation microsegregation occurs, leading to domains rich in one component and pure in the other(s).

The simplest case is that of binary copolymers, where the chains only contain two distinct types of monomers $(A$ and $B)$. According to the way in which both species are distributed along the chain, one can find several patterns. These include (see Fig. 1.1):

a) diblock copolymers (...AAAAABBBBB...), which are formed by two connected blocks, each block being enterely constituted by $A$ - or by $B$-monomers.

b) gradient copolymers, in which the population of monomers of a given species exhibits a gradual change (increase or decrease) from one side of the chain to the other, described by a distribution function $p(i)$, with $i$ denoting the monomer position along the chain. Obviously, the other species follows the complementary distribution $1-p(i)$. Diblock copolymers are a particular case of gradient copoly- 


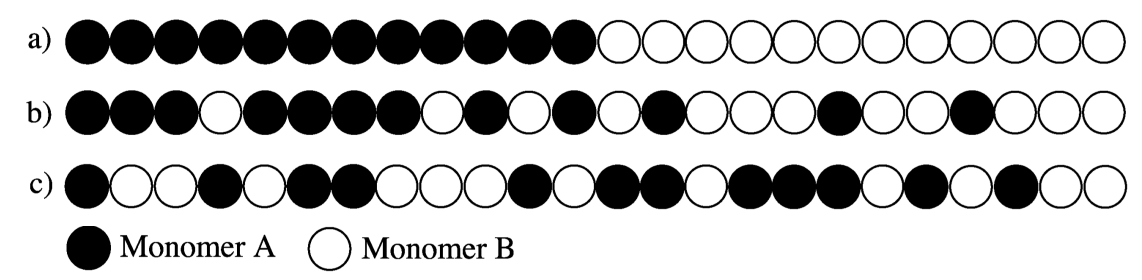

Figure 1.1: Examples of different patterns for the distribution of monomers in binary copolymer chains. a) diblock, b) gradient, c) random.

mers when $p(i)$ is a step function.

c) random copolymers, in which monomers of both species are randomly distributed along the chain.

Other patterns include alternating copolymers, formed by regularly alternating pure

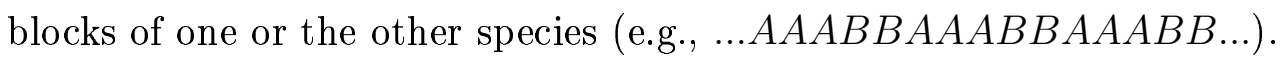

Except in the case of purely random copolymers, the segregated domains induced by the former patterns can be arranged leading to long-range ordered phases with regularly spaced domains $[1,2,3]$. The transition from the disordered to the ordered state can be driven by tuning the relevant control parameters, i.e., for sufficiently strong uncompatibility of the components (e.g., by decreasing temperature), not too asymmetric compositions, and sufficiently long blocks or chains. The transition between the different ordered mesophases can be driven by tuning the fraction of each component. Thus, for simple linear diblock copolymers the sequence of stable phases includes, from asymmetric to symmetric composition, spherical, cylinder, gyroid, and lamellar morphologies (see the phase diagram in Fig. 1.2).

Self-assembly of block copolymers is one of the most active fields of research in soft matter systems $[1,2,3,4,5,6]$. Ordered mesophases of block copolymers have numerous advanced technological applications [7], as membranes, photonic materials or templates for nanostructured systems.

In this work we focus on the case of simple linear diblock copolymers $(A B)$. Copolymers of very well defined diblock architecture can be obtained by techniques like termination-free anionic polymerization [8]. The control parameters determining the phase behaviour (Fig. 1.2) are the total degree of polymerization $N$, the 

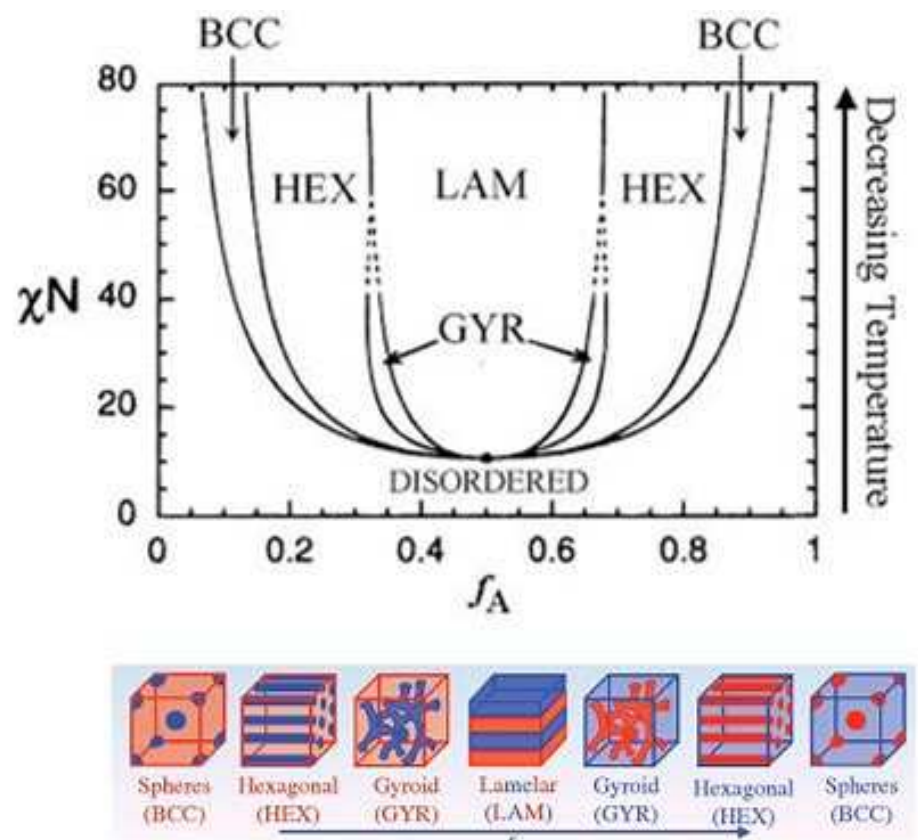

Figure 1.2: Phase behaviour of diblock copolymers [9].

composition $f$ (fraction of each species), and the $A-B$ interaction parameter $\chi$ (Flory-Huggins parameter) [2]

$$
\chi=\frac{1}{k_{B} T}\left\{\epsilon_{A B}-\frac{1}{2}\left(\epsilon_{A A}+\epsilon_{B B}\right)\right\}
$$

where $\epsilon_{\alpha \beta}$ is the interaction energy between $\alpha$-and $\beta$-monomers. If $\chi$ is negative, the mixing is favourable while in the case $\chi>0$ the cross-interaction is unfavourable, and in concentrated solutions or melt states the blocks are strongly segregated. Segregation and eventually microphase separation in ordered structures emerges as a way of minimizing the free energy of the system. Thus, the loss of entropy originating from the segregation is largely compensated by reducing the energetically unfavourable $A-B$ contacts.

We can distinguish two limit cases in the ordered state [1]. In the weak segregation limit (WSL), close the order-disorder transition (ODT), which for $f \sim 0.5$ occurs at $\chi N \sim O(10)$, the conformations of the blocks remain Gaussian as in a disordered melt. Thus their average end-to-end radius scales as $N^{1 / 2}$, with $N$ the 


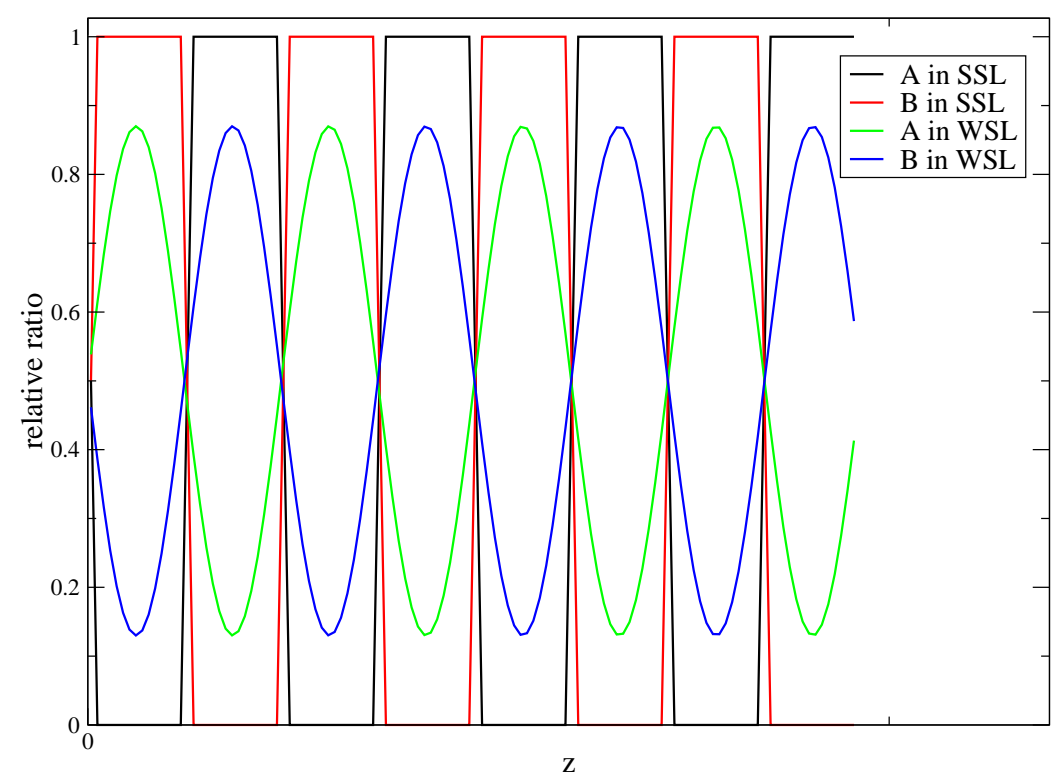

Figure 1.3: Density profile of a lamellar phase $(f=0.5)$ in the SSL and WSL.

number of monomers per block. Likewise, the domain size scales as $d \sim N^{1 / 2}$. For lamellar phases in the WSL the composition profile is approximately sinusoidal (Fig. 1.3). In the strong segregation limit (SSL, for $\chi N>O(100)$ ), narrow interfaces of width $\sim \chi^{-1 / 2}$ separate well defined $A$ - and $B$-domains. The system tends to reduce the energetically unfavourable $A-B$ contacts by minimizing the interfacial area, at the expense of an entropic penalty. The latter results from the necessary stretching of the blocks, leading to strongly perturbed non-Gaussian conformations. As a consequence of chain stretching, in the SSL the size of the lamellar domains scales as $d \sim N^{2 / 3} \chi^{1 / 6}$.

The phase boundaries of Fig. 1.2 and the former scaling properties are derived within simplified lattice mean-field theories. More elaborated self-consistent field theories $[10,11,12,13]$ lead to modified values of $\chi N$ and $f$ for the phase boundaries, and predict additional complex phases in narrow regions of the phase diagram. Having said this, the qualitative picture of Fig. 1.2 is still correct. 


\subsection{Dynamics in block copolymers}

Several dynamic features in ordered phases of diblock copolymers have been investigated in cylinder $[5,14,15,16,17]$, gyroid [5, 18], spherical [19, 20, 21, 22, 23, $24,25,26]$, and mostly in lamellar morphology [6, 14, 18, 27, 28, 29, 30, 31, 32, $33,34,35,36,37,38,39,40,41,42,43,44,45,46]$. In lamellae-forming systems the diffusivity exhibits a bifurcation, around the order-disorder transition, in two components parallel and perpendicular to the lamellar planes. This bifurcation is smooth and the diffusivity shows no discontinuity. For the case of short nonentangled chains, diffusion in the lamellar phase is strongly anisotropic, with a strong reduction of the perpendicular component [29, 37, 38, 39]. On the other hand, for long entangled chains differences between perpendicular and parallel diffusivities are strongly reduced $[6,30,31,40]$.

Concerning the local segmental dynamics i.e., the structural $\alpha$-relaxation associated to the glass transition $[47,48]$, a general observation is that the $\alpha$-relaxation times in the lamellar phase are close to those of the corresponding homopolymer $[35,41,43,44,45]$. Usually, the former are slightly shifted to shorter or longer values than in the homopolymer if the segmental dynamics of the other block is respectively faster or slower, i.e., if the other block has a lower or higher glass transition temperature $T_{\mathrm{g}}$. Despite the similar values of the $\alpha$-time scales, the dynamic response in the lamellar phase exhibits a strong broadening in the low-frequency side (long times) in comparison with the corresponding homopolymer. This effect is particulary visible by means of broadband dielectric spectroscopy (BDS) [41, 43, 45], which reveals a progressive broadening by several frequency decades on decreasing temperature. This feature is usually rationalized in terms of dynamic heterogeneity. It is assumed that the segmental relaxation in the center of the lamellar domains is esentially the same as in the corresponding homopolymer. However the former is strongly perturbed as the lamellar interface is approached, leading to gradients of mobility [43]. This proposed microscopic picture has connections with the scenario observed for dense polymer brushes [49, 50], polymer melts confined in slits [51, 52], 
free-standing or supported polymer thin films [53, 54, 55, 56], and more generally, with dynamic features of the structural relaxation in confined fluids $[57,58,59]$.

\subsection{Motivations of this work}

In this work we present a detailed characterization of the heterogeneity of the segmental dynamics in lamellar phases. We study this feature by means of molecular dynamics simulations of a generic bead-spring model of diblock copolymers. Aiming to mimic usual experimental systems [27, 28, 35, 42, 43, 45], the corresponding homopolymers exhibit very different intrinsic mobilities, i.e., different glass transition temperatures. The simulations are performed in the strongly segregated regime, with a well defined lamellar morphology. We characterize the dynamic heterogeneity as a function of both the monomer location along the chain and the distance to the nearest interface between consecutive domains. Both characterizations of the dynamic heterogeneity reveal moderate gradients of mobility, of about one time decade, in the investigated temperature range, which qualitatively probes relaxation time scales of up to hundreds nanoseconds. However, the extrapolation of the results to lower temperatures leads to an increasing spread over several time decades. This spread originates mostly from monomers located at the inmediate neighborhood of the interface. Beyond such distances the structural relaxation approaches that of the homopolymer. Thus, we conclude that the observed dynamic heterogeneity is esentially an interfacial effect. It does not originate from gradients of density over the domains. Indeed such gradients are absent and the local density within the domains is identical to that of the corresponding homopolymers. 


\section{Chapter 2}

\section{Simulations}

\subsection{Background}

Since its birth in the early 50's [60,61], molecular simulation has become a powerful technique for investigating microscopic properties in many areas of condensed matter physics, chemical physics or biophysics. See, e.g., the reference books [62, 63, 64]. Simulations provide a direct calculation, from the atomic trajectories, of observables that cannot be accessed in experiments or that are indirectly obtained from them on the basis of approximations or model assumptions.

The manner in which the system is propagated during the simulation defines two main kinds of molecular simulation techniques: molecular dynamics (MD) and Monte Carlo (MC) methods. In MD simulations the atoms are propagated by numerical solution of the corresponding classical equations of motion with small time steps. The evolution can be fully deterministic (as for purely Newtonian dynamics) or can incorporate some additional stochastic character (as for Langevin or Brownian dynamics, frequently used to describe implicitly contributions from a fast solvent). In MC simulations the atoms are propagated through unphysical random motions, which are accepted or rejected according to energetic criteria. Generally speaking, MD simulations are adequate to determine dynamic properties of the system (diffusivities, van Hove functions...). When the involved time scales are accessible, MD can also be used for equilibration of the system, generating configurations from 


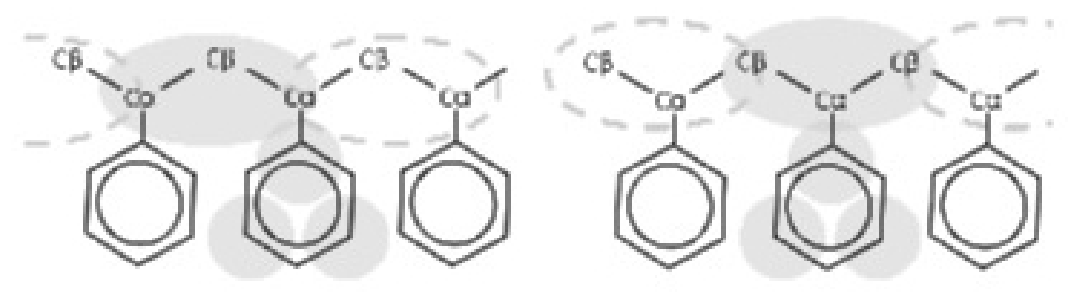

Figure 2.1: Two coarse-graining schemes for polystyrene [68].

which static observables (radial distribution functions, form factors...) can be calculated. Static properties can also be investigated by $\mathrm{MC}$ simulations. Indeed many different and sophisticated MC techniques have been developed for studying phase behaviour or equilibration of large-scale static properties which cannot be achieved by standard MD or MC in accessible time scales. Concerning dynamic properties, $\mathrm{MC}$ is in general reliable, through some conversion factor to MD time scales, in time windows governed by random-walk behaviour (e.g., diffusive regimes). However care must be taken when comparing $\mathrm{MC}$ with real dynamics at short and intermediate time scales.

\subsection{Coarse-grained simulations of polymer systems}

The main limitations of simulation techniques are given by the accuracy of the used force field and by the computational resources. Even by parallel computation in standard modern machines, it is very demanding to simulate dense polymer systems with fully atomistic force fields (i.e., incorporating the whole chemical structure of the monomeric units) for, e.g., time scales of 100 nanoseconds and cell dimensions of $50 \AA$. This constitutes a clear limitation for the case of nanostructured phases, as the lamellar phases of diblock copolymers investigated in this work.

Aiming to extend the simulation scale to longer times and larger cells, the investigated system can be coarse-grained at different levels. The coarse-graining procedure consists of reducing the number of coordinates of the system by substituting 
the original polymer chains by chains of 'superatoms', leading to a so-called 'united atom' (UA) model $[65,66,67,68]$. The coordinates of each superatom represent the center-of-mass of a given set of real atoms (e.g., 2-3 carbons and the hydrogens bonded to them). See Fig. 2.1 for an example of coarse-graining schemes in polystyrene [68]. The next step is to derive effective potentials between superatoms. Such potentials are parametrized, in general by following some iterative procedure, in order to optimize the quantitative description of some equilibrium microscopic or macroscopic property (e.g, static correlations previously obtained in atomistic simulations, experimental densities, experimental radii of gyration...). Obviously, static and dynamic properties at the coarse-grained scales (e.g., fast vibrations) cannot be captured by the coarse-grained force field.

However, due to the reducion in the number of coordinates (from several atoms to a single superatom), the coarse-grained model allows to explore larger time and length scales than those reached by fully atomistic simulations. This is also favoured by the much more simple form of coarse-grained force fields in comparison with fullyatomistic ones, even for a low level of coarse-graining (2-3 carbons per superatom). Thus, coarse-grained force-fields usually consist of a Lennard-Jones term describing non-bonded interactions, and a bonding potential for connected superatoms. In general they also include bending potentials (dependent on the bending angle between consecutive bonds) and torsion potentials (dependent on the angle between dihedral planes). The two latter terms describe the intrinsic stiffness of the chain backbone, which leads to local deviations from the large-scale Gaussian chain conformations.

A further level of coarse-graining consists of neglecting bending and torsion terms, retaining just the ingredients of excluded volume (non-bonded Lennard-Jones interactions) and connectivity (bonding potentials) in a schematic bead-spring model for the chain backbone (see below). Originally introduced in the 80's by Grest and Kremer [69], the bead-spring model is widely used by the computational community of polymer physics in many different topics of research. These include complex physical situations as e.g., micellization, mesoscopic self-assembly, adsorption at interfaces, chemical gelation or translocation. In general simple bead-spring models 
cannot provide an accurate quantitative description of properties of real polymers (fully-atomistic or specifically designed UA models are used for that). Actually simple bead-spring models are aimed to test theories, general hypothesis, and to predict generic behaviours, which are weakly dependent on the chemical composition and are just controlled by some basic ingredients (chain length, mixture composition, uncompatibility of components, macromolecular architecture...). Having said this, units of bead-spring models can be qualitatively mapped to real units [70] (see below). Note that indeed the functional forms of quantitative UA-models and simple bead-spring models are not very different.

\subsection{Model and simulation details}

Aiming to understand generic trends of dynamics in lamellar phases, we have used a bead-spring model for diblock copolymers. We simulate polymer melts of identical bead-spring chains of $N=350$ monomers. The simulation box contains $N_{\text {cha }}=136$ chains. The diblock systems have symmetric composition. Thus, each diblock chain consists of $N_{\mathrm{F}}=N_{\mathrm{S}}=175$ monomers of, respectively, the species F ('fast') and $\mathrm{S}$ ('slow'). The interaction between any two given monomers of the species $\alpha$ and $\beta$ $\in\{\mathrm{F}, \mathrm{S}\}$ is given by a shifted Lennard-Jones potential:

$$
V_{\alpha \beta}(r)=4 \epsilon_{\alpha \beta}\left[\left(\frac{\sigma_{\alpha \beta}}{r}\right)^{12}-\left(\frac{\sigma_{\alpha \beta}}{r}\right)^{6}+\frac{1}{4}\right]
$$

for $r<r_{\mathrm{c}}$ and $V_{\alpha \beta}(r)=0$ for $r \geq r_{\mathrm{c}}$. By using a value $r_{\mathrm{c}}=2^{1 / 6} \sigma_{\alpha \beta}$ for the cut-off distance, the potential is purely repulsive and has no local minima. Moreover potential and forces are continuous at $r_{\mathrm{c}}$. In addition to the Lennard-Jones potential, two connected monomers in a same chain interact through a finitely extensible nonlinear elastic potential (FENE) [70]:

$$
V_{\alpha \beta}^{\mathrm{FENE}}(r)=-\epsilon_{\alpha \beta} K_{\mathrm{F}} R_{0}^{2} \ln \left[1-\left(\frac{r}{R_{0} \sigma_{\alpha \beta}}\right)^{2}\right],
$$

with $K_{\mathrm{F}}=15$ and $R_{0}=1.5$. Eqs. (2.1) and (2.2) define the Grest-Kremer model for simulation of polymer systems. The sum of the potentials (2.1) and (2.2) yields an 


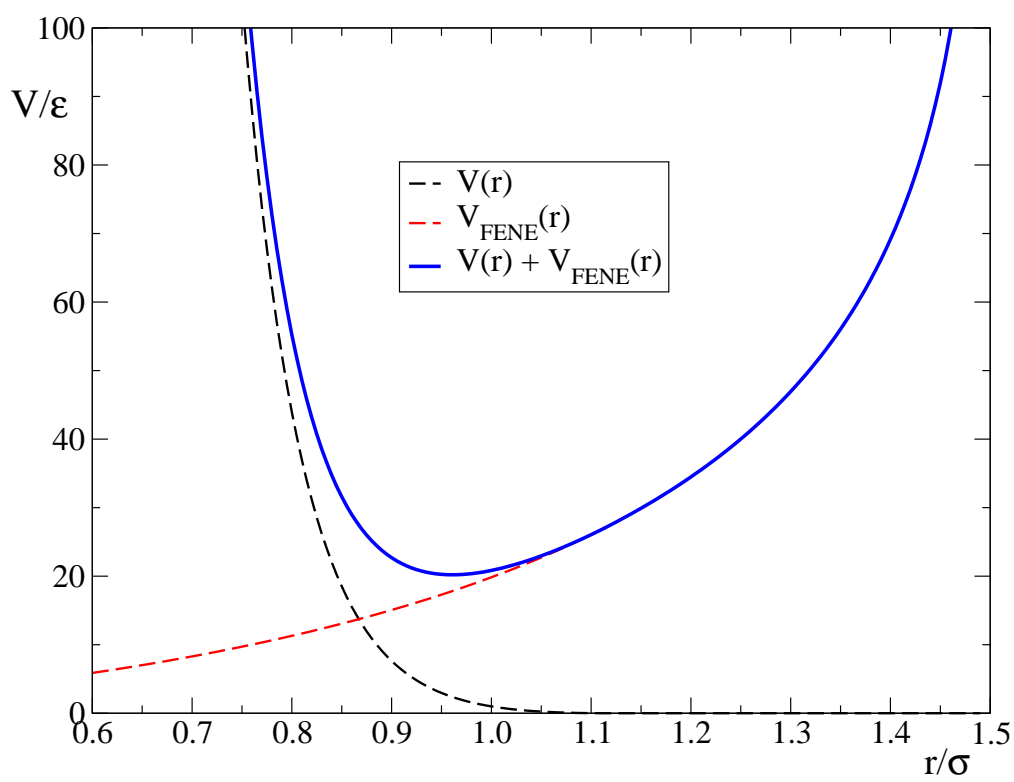

Figure 2.2: Potentials for non-bonded and bonded interactions, and effective potential between connected monomers.

effective potential (see Fig. 2.2) between connected monomers which shows a sharp minimum at $r=0.96 \sigma_{\alpha \beta}$ and guarantees chain uncrossability [70]. We use identical monomer masses $m_{\mathrm{F}}=m_{\mathrm{S}}=m=1$ and interaction diamaters $\sigma_{\mathrm{FF}}=\sigma_{\mathrm{SS}}=\sigma_{\mathrm{FS}}=$ $\sigma=1$. In order to obtain different mobilities for the two species, we use different values for the energy scale of the self-interaction, $\epsilon_{\mathrm{FF}}=0.35$ and $\epsilon_{\mathrm{SS}}=1$, which leads to faster dynamics for the F-component (see Chapter 3). For the cross-interaction we use $\epsilon_{\mathrm{FS}}=9$. With this strong energetic penalty for the F-S interactions, the diblock system is expected to be in the strongly segregated regime at temperatures of interest [39], as we confirm below.

In the following, temperature $T$, pressure $P$, monomer number density $\rho$, time $t$, and distance will be given respectively in units of $\epsilon_{\mathrm{BB}} / k_{B}$ (with $k_{B}$ the Boltzmann constant), $\epsilon_{\mathrm{BB}} \sigma^{-3}, \sigma^{-3}, \sigma\left(m / \epsilon_{\mathrm{BB}}\right)^{1 / 2}$, and $\sigma$. Simulation units of the Grest-Kremer model for distance and time can be qualitatively mapped to real units as $\sigma \sim 5$-10 $\AA$ and $\sigma(m / \epsilon)^{1 / 2} \sim 1-10$ ps (see the discussion in, e.g., Refs. [51, 70]).

We also simulate the corresponding homopolymer systems of F- and S-monomers. For these we use a cubic simulation box with periodic boundary conditions. Com- 
putation time is reduced by using a linked-cell method [63] for calculation of interparticle distances within interaction ranges. We perform an initial run in the isothermal-isobaric $(N P T)$ ensemble at external pressure $P=3.0$, by using the Nosé-Hoover algorithm [63]. The corresponding equations of motion are:

$$
\begin{gathered}
\dot{\mathbf{r}}_{i}=\frac{\mathbf{p}_{i}}{m_{i}}+\frac{p_{\epsilon}}{W} \mathbf{r}_{i} \\
\dot{\mathbf{p}}_{i}=\mathbf{F}_{i}-(1+1 / N) \frac{p_{\epsilon}}{W} \mathbf{p}_{i}-\frac{p_{\xi}}{Q} \mathbf{p}_{i} \\
\dot{V}=3 V p_{\epsilon} / W \\
\epsilon=\ln (V / V(t=0)) \\
\dot{p}_{\epsilon}=3 V\left(P_{\mathrm{int}}-P\right)+\frac{1}{N} \sum_{i=1}^{N} \frac{p_{i}^{2}}{m_{i}}-\frac{p_{\xi}}{Q} p_{\epsilon} \\
P_{\mathrm{int}}=\frac{1}{3 V} \sum_{i=1}^{N}\left(\frac{p_{i}^{2}}{m_{i}}+\mathbf{r}_{i} \cdot \mathbf{F}_{i}\right) \\
\dot{\xi}=p_{\xi} / Q \\
\dot{p}_{\xi}=\sum_{i=1}^{N} \frac{p_{i}^{2}}{m_{i}}+\frac{p_{\epsilon}^{2}}{W}-(3 N+1) k_{\mathrm{B}} T
\end{gathered}
$$

In these equations $\mathbf{r}_{i}, \mathbf{p}_{i}$ and $m_{i}$ are the coordinates, momenta, and masses of the $N$ particles of the system. $\mathbf{F}_{i}$ is the force (derived from Eqs. (2.1) and (2.2)) on the $i$ th-particle. $P$ and $T$ are the requested pressure and temperature. $P_{\text {int }}$ is the internal virial pressure. $V$ is the volume of the simulation cell. The coordinates, $\xi$ and $\epsilon$, of two 'abstract' particles (thermostat and barostat respectively) appear in the equations of motion. The respective momentum and mass are $p_{\xi}$ and $Q$ for $\xi$, and $p_{\epsilon}$ and $W$ for $\epsilon$. It can be demonstrated that the ensemble average in the former extended system $\left(\left\{\mathbf{r}_{i}, \mathbf{p}_{i}, \xi, p_{\xi}, \epsilon, p_{\epsilon}\right\}\right)$ is equivalent to the average of the real system $\left(\left\{\mathbf{r}_{i}, \mathbf{p}_{i}\right\}\right)$ in the NPT ensemble [63].

After this NPT run, the thermostat and barostat particles are removed, and a further equilibration run is performed in the real system (under the Hamilton's equations of motion, $\dot{\mathbf{r}}_{i}=\mathbf{p}_{i} / m_{i}, \dot{\mathbf{p}}_{i}=\mathbf{F}_{i}$ ) at constant volume. The system is thermalized by periodic velocity scaling, in order to keep the kinetic energy fluctuating around the equilibrium value $3 N k_{\mathrm{B}} T / 2$. Finally, a run is performed in the 
microcanonical ensemble for production of configurations, from which we compute the static and dynamic observables presented below. Equations of motion are integrated in the Martyna's scheme $[63,71]$ for the NPT runs and in the velocity-Verlet scheme [63] for the other runs. For the $N P T$ runs we use values $W, Q \sim 3 N k_{\mathrm{B}} T$ for the masses of the barostat and thermostat. With this choice, the kinetic energy and virial pressure remain stable with the expected small fluctuations around $3 N k_{\mathrm{B}} T / 2$ and $P$ respectively.

For all runs we use typical integration time steps of $2 \times 10^{-3} \leq \delta t \leq 4 \times 10^{-3}$, according to the investigated temperature. The latter covers the range $0.14 \leq$ $T \leq 0.30$. The corresponding equilibrium densities for $P=3.0$ cover the range $1.06 \geq \rho \geq 0.98$ and $1.00 \geq \rho \geq 0.92$ for respectively the F- and S-homopolymer. These are typical melt densities for similar bead-spring models of polymer systems $[51,70]$.

A different equilibration procedure is needed in the case of the lamellar phase of the diblock system. Except for rather short chains at high temperature, the spontaneous formation in the melt state of well-ordered mesophases, by starting from a disordered configuration, is unfeasible within simulation time scales [25, 34, 39, 72]. Thus, spontaneous evolution generally leads to a metastable microsegregated phase, which may exhibit the expected geometry of the segregated domains at short and even middle length scales, but lacks of large-scale order [25, 34]. The usual strategy for obtaining ordered phases is actually to equilibrate the system by starting from a configuration with the expected geometry [39, 73, 74, 75]. Thus, we have followed a procedure based on the method proposed by Schultz et al. [75]. The simulation cell is orthoedric, of sides $L_{x}=L_{y} \neq L_{z}$, with $z$ the coordinate perpendicular to the lamellar planes. We first set four equidistant F-S interfaces in the simulation cell. Then, starting from the paralell planes representing the interfaces, S- and F-blocks are constructed by chain grow-up. Thus, all the S-blocks linked to a same interface grow up over the same $z$-direction, and the respective F-blocks grow up over the opposite one. Blocks of the same species and linked to consecutive interfaces grow up over opposite directions, leading to a sequence of blocks SF-FS-SF-FS... along 
the $z$-direction (see Figure 3.1). A constraint is imposed in the growth-up procedure in order to avoid core overlap of new monomers with previously inserted ones. The same number of chains, $N_{\text {cha }} / 4$, is constructed at each interfacial plane.

We select a volume $V=L_{x} L_{y} L_{z}$ for the initial simulation box in order to get an initial density $\rho=N_{\text {cha }} N / V \sim 0.5$. Then we start a run in the NPT ensemble as described above, at the same $P=3.0$. Since volume fluctuations are isotropic in this method (i.e, the three cell axes are rescaled by the same factor [63]), it is clear that the correct equilibrium lamellar spacing can only be achieved fortuitously. Thus, isotropic volume fluctuations will in general produce an unbalance between the components of the internal pressure, $\left\langle P_{x}\right\rangle=\left\langle P_{y}\right\rangle \neq\left\langle P_{z}\right\rangle$. In order to balance the three components we periodically apply a box length search algorithm [75], which maintains the instantaneous volume, but rescale the $z$-coordinate by a factor $1-|\xi| \leq f \leq 1+|\xi|$, and simultaneously the $x, y$-coordinates by a factor $f^{-1 / 2}$. We use $f>1$ when $\left\langle P_{x, y}\right\rangle^{\prime}<\left\langle P_{z}\right\rangle^{\prime}$ and $f<1$ when $\left\langle P_{x, y}\right\rangle^{\prime}>\left\langle P_{z}\right\rangle^{\prime}$. Here $\langle\ldots\rangle^{\prime}$ denotes average over a certain interval prior to the next rescaling. We initially take $|\xi| \lesssim 0.01$ and progressively decrease its value as the pressure balance $\left\langle P_{x}\right\rangle=$ $\left\langle P_{y}\right\rangle=\left\langle P_{z}\right\rangle=P$ is approached. Once this is achieved we perform, as described above, a further equilibration run at constant volume under periodic velocity scaling, and a final production run in the microcanonical ensemble. We investigate the same temperature range $0.14 \leq T \leq 0.30$ as for the homopolymers. The obtained equilibrium densities of the lamellar domains are equal, within statistics, to those of the respective homopolymers (see Chapter 3 ).

All the simulations have been performed with a home-made code. Typical simulation times for both equilibration and production runs are of 30 million integration time steps. In absolute units the latter typically cover up to time scales of $t \sim 10^{5}$, which qualitativey correspond in real systems to several hundreds nanoseconds (see above). Static profiles (see Chapter 3) are averaged over configurations at typically 100 equispaced times. Dynamic observables are averaged over 20 equispaced time origins. 


\section{Chapter 3}

\section{Results}

\subsection{Static properties}

Table 3.1 shows at the different investigated temperatures the size of the lamellar domains, estimated as $L_{z} / 4 \sigma$, with $L_{z}$ the size of the simulation cell along the $z$ coordinate perpendicular to the lamellar planes. As expected, a progressive increase of the domain size is observed by decreasing temperature.

The top panel of Figure 3.1 shows a typical snapshot of the lamellar phase. The combination of isotropic box fluctuations with periodic anisotropic scaling (see Chapter 2) produces structures with well-defined order. We obtain stable ordered lamellar structures at all the investigated temperatures. The bottom panel of Figure 3.1 shows two typical conformations of the F-blocks, extending over a same F-domain and linked to different S-domains. The blocks do not show gaussian conformations but are strongly stretched. In spite of the associated entropic penalty, stretched conformations are favoured by the strong reduction of energetically un-

\begin{tabular}{llllll}
\hline \hline$T$ & 0.30 & 0.22 & 0.17 & 0.15 & 0.14 \\
\hline$L_{z} / 4 \sigma$ & 29.95 & 32.22 & 33.24 & 33.77 & 33.96 \\
\hline
\end{tabular}

Table 3.1: Lamellar spacing at the different investigated temperatures. 

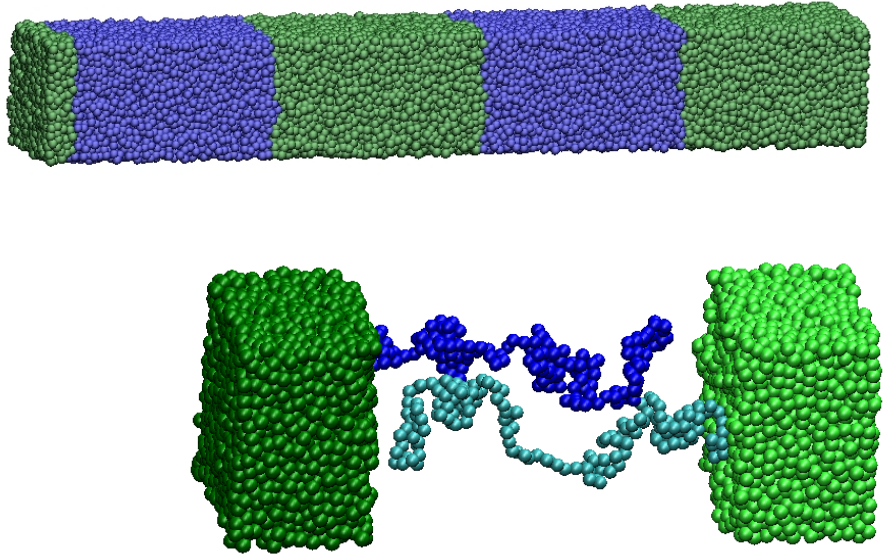

Figure 3.1: Top: typical snapshot of the lamellar phase. F- and S-monomers are represented respectively in blue and green. Bottom: Typical conformations of two F-blocks (blue, center of the figure). The S-domains (green) to which they are linked are partially represented at the left and right sides of the figure. Light and dark F-blocks are respectively linked to light and dark S-domains.

favourable contacts between distinct blocks. This is a well-known feature for strongly segregated lamellae $[27,28,73,76]$.

We characterize local density profiles of each species (F and S) along the direction perpendicular to the lamellar planes. For this we introduce the quantity $\rho(z)=$ $n(z) /\left[L_{x} L_{y} \delta\right]$, with bin size $\delta=0.1 \sigma$ and $n(z)$ the number of monomers of the considered species which are located in the interval $(z-\delta / 2, z+\delta / 2)$. Figure 3.2 displays the local density profile at $T=0.15$. Sharp boundaries are observed between the F- and S-domains, as expected for the strongly segregated regime. The domain size at $T=0.15$ is about $34 \sigma$, which qualititatively corresponds to about $15-35$ $\mathrm{nm}$ in real systems (see Chapter 2). As observed in previous investigations on strongly segregated lamellar phases [38, 39], the local density profiles exhibit, within statistics, a flat structure from the inmediate neighborhood of the interface to the center of the domains (see Figure 3.2b). This is similar to the observation in freestanding films $[54,56,77,78]$. It is instead rather different from the layering behavior observed near rigid smooth walls, for both free $[51,52,56,78]$ and grafted chains 
$[79,80]$. There, the local density profiles exhibit strong oscillations along the $z$ direction, of increasing amplitude on approaching the wall. As in free-standing films, the mobile and irregular character of the interface seems to frustrate layering in the lamellar domains, leading to the observed flat profiles in Figure 3.2.

With the used normalization for $\rho(z)$ (see above), we can directly estimate differences between the local density within the domains and the macroscopic density $\rho_{\text {hom }}$ of the respective homopolymers. This comparison is shown in detail in Figure $3.2 \mathrm{~b}$. We define the $z$-position of the interface, $z_{\text {int }}$, as the crossing point of the density profiles $\rho(z)$ of the respective F- and S-domains. We only observe clear differences between $\rho(z)$ and $\rho_{\text {hom }}$ in the inmediate neighborhood of the interface, at distances $\Delta z=\left|z-z_{\text {int }}\right| \lesssim 3 \sigma$. By mapping to real units (see Chapter 2) the latter corresponds to an interfacial half-width of about $1.5-3 \mathrm{~nm}$, in qualitative agreement with experimental values in strongly segregated phases $[26,35,43]$. In the rest of the domain we find that, within statistics, $\rho(z)=\rho_{\text {hom }}$. Therefore, Figure 3.2 clearly demonstrates that gradients of density (defined as variations of $\rho(z)$ along the domains) are absent, and that the density of each homopolymer is unperturbed in the lamellar phase of the diblock system.

Now we identify the type of monomers located near the interfaces. We label the monomers along the chain as $i=1,2, \ldots N$, from one chain end to the other one, and starting from the F-end. By defining the interface positions $z_{\text {int }}$ as above, we can obtain the distribution $p(i)$ of interfacial monomers. For this we compute the histogram of $i$-monomers which are at a distance $\Delta z<2 \sigma$ from the nearest interface. Results are presented in Figure 3.3. Not surprisingly, $p(i)$ displays a sharp maximum around $N / 2=175$. This just reflects the fact that monomers close to the F-S bond (in the following denoted as junction monomers) are preferently located in the inmediate neighborhood of the interface. There are other types of monomers which also follow the criterium $\Delta z<2 \sigma$. Such monomers are those close to the block ends (in the following denoted as end monomers), as indicated (see Figure 3.3) by the two secondary peaks of $p(i)$ in the ranges $i \lesssim 50$ and $i \gtrsim 300$. The presence of these secondary peaks is easily understood by inspection of the 

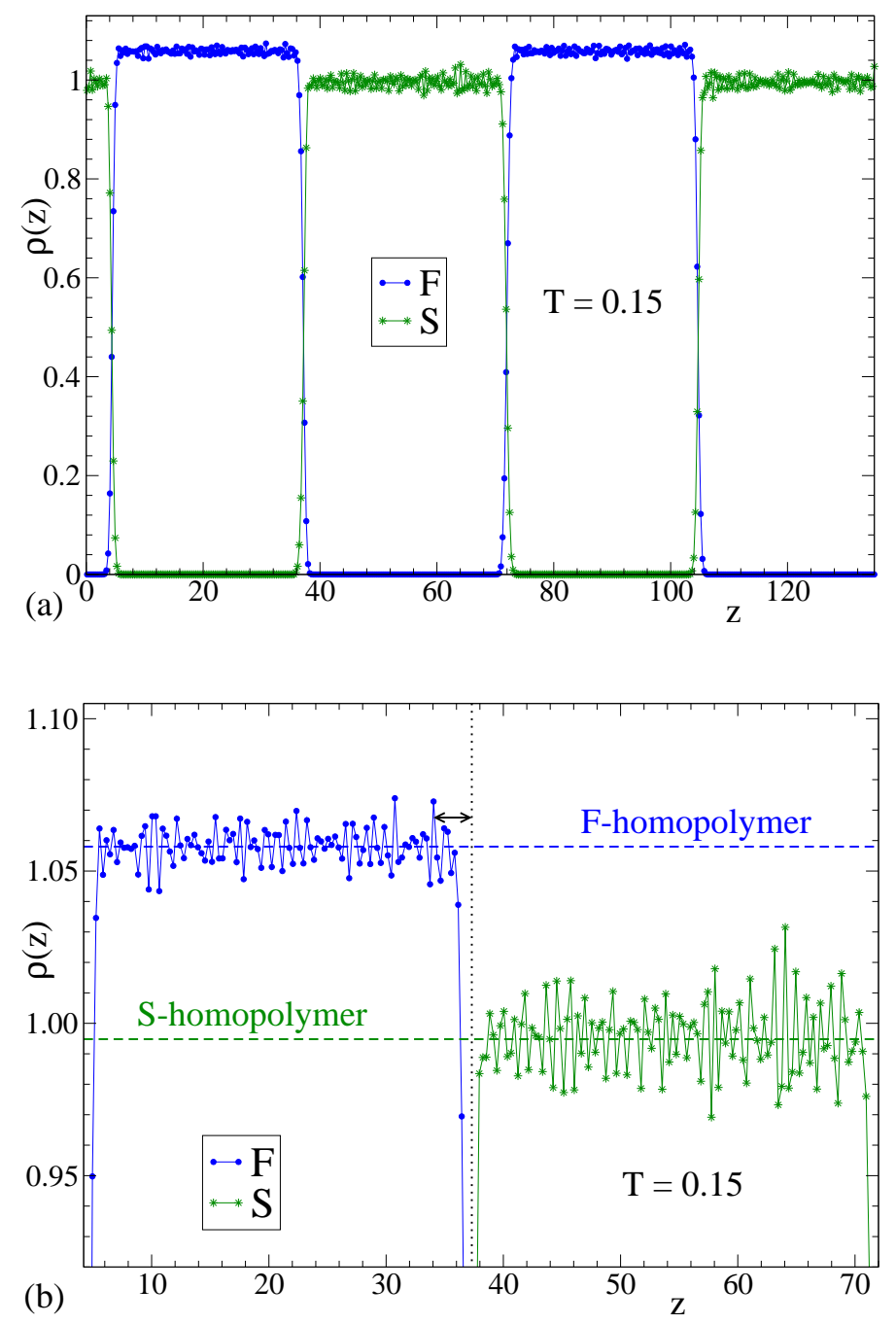

Figure 3.2: Panel (a): Local density profiles $\rho(z)$ for the F- and S-monomers in the direction perpendicular to the lamellar planes, at $T=0.15$. Panel (b): For clarity, data of panel (a) are shown only for two selected layers. Also included are the macroscopic densities $\rho_{\text {hom }}$ of the F- and S-homopolymers (thick dashed lines) at the same $T$. The vertical dotted line indicates the interface position. The arrow extends from the interface to a distance $\Delta z=3 \sigma$. 


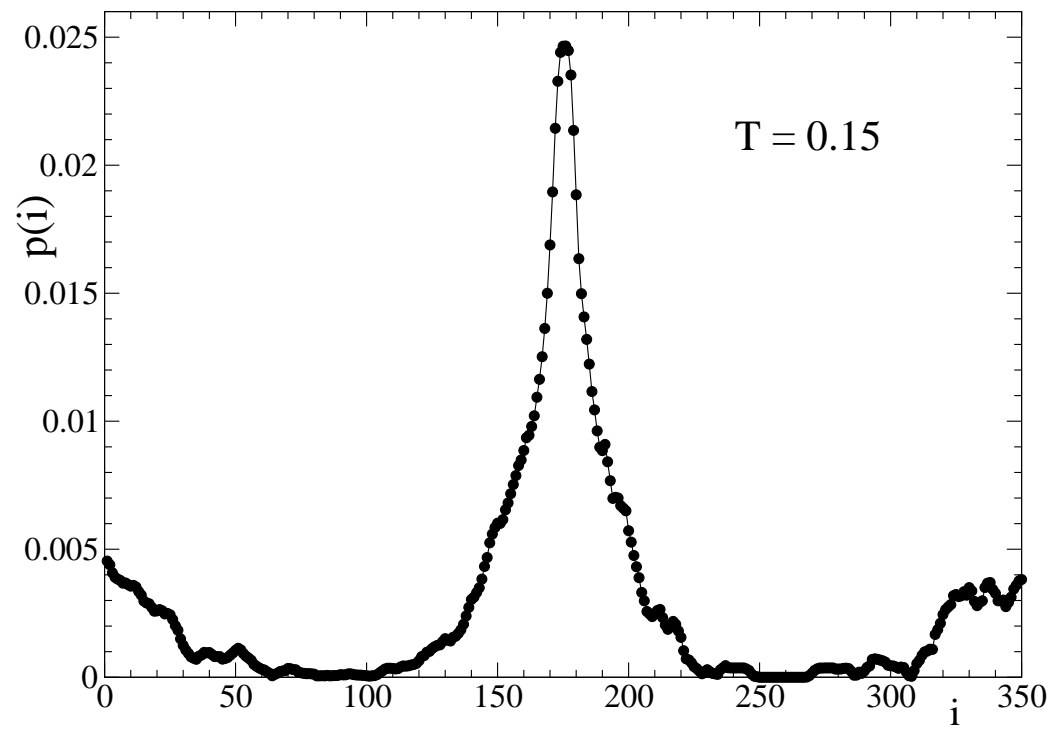

Figure 3.3: Distribution of interfacial monomers (see text for definition) at $T=0.15$.

bottom panel of Figure 3.1, which shows that stretched blocks extend from one interface to the next one. The contribution of end monomers to the total population of interfacial monomers is significant. Indeed, we estimate such a contribution, by simple integration of $p(i)$, as about a $25 \%$ of the total. This result is consistent with previous observations in strongly segregated lamellar phases [39].

\subsection{Dynamics}

Now we discuss dynamic features in the lamellar phase. Panel (a) of Figure 3.4 displays results for the $T$-dependence of the mean squared displacement, $\left\langle\Delta r^{2}(t)\right\rangle$, of F- and S-monomers in the diblock system. The corresponding results for the orientational correlator, $P(t)$, of F-F and S-S bonds are shown in panel (b). The orientational correlator is defined as $P(t)=\langle\cos \theta(t)\rangle$, with $\theta(t)$ the angle between the orientations of the bond at $t=0$ and at the considered $t$. As usual in twocomponent polymer systems [81], a progressive dynamic separation between the F- and S-components is observed as temperature decreases. Estimating structural relaxation times $\tau$ as those for which $\left\langle\Delta r^{2}(\tau)\right\rangle=\sigma^{2}$ or $P(\tau)=e^{-1}$, we observe a separation of about one decade in $\tau$ at the lowest investigated $T=0.14$ (see 
Figure 3.4). For comparison we have included the corresponding data for the F- and S-homopolymers at the two lowest investigated temperatures $T=0.14$ and $T=0.15$. Small differences are observed between the blocks and their respective homopolymers (also for the data at the other temperatures, not shown for the homopolymers). The coupling to a block of lower or higher intrinsic mobility has a different effect on the Fand S-components. Thus, the segmental relaxation in the F-blocks is slightly slower than in the F-homopolymer, in agreement with the usual experimental observation in blocks coupled to a slower component $[35,41,43,44,45]$. In the case of the S-blocks the opposite effect is observed for the mean squared displacement, which is slightly sped up in comparison with that of the S-homopolymer. However no clear differences are observed between the orientational correlators of the S-blocks and the S-homopolymer.

After the initial ballistic regime a plateau arises in both $\left\langle\Delta r^{2}(t)\right\rangle$ and $P(t)$, at $t \approx 1$, for $T<0.22$. The plateau extends over longer time scales as temperature decreases. This feature is a well-known manifestation of the caging regime, i.e., the temporary trapping of each particle by the surrounding ones, which is characteristic of glass-forming liquids on approaching the glass transition temperature [47, 48]. The plateau regime (or the ballistic one at high $T$ ) is not followed by a crossover to an ultimate diffusive regime $\left\langle\Delta r^{2}(t)\right\rangle \sim t$. It is well known that prior to it, homopolymers exhibit subdiffusive behavior over several time decades, corresponding to Rouse-like dynamics of the internal chain degrees of freedom [82]. Even further subdiffusive regimes, usually assigned to reptation dynamics [82], arise in the case of long chains as those simulated here, of length $N=350$ beyond the entanglement value $\left(N_{\mathrm{e}} \sim 60\right.$ for bead-spring models [83]). How the former pictures for chain dyamics are affected in the lamellar phase, and in particular the effect of the coupling of each block to other with very different mobility, are questions which are beyond the scope of this work and will be addressed in the future.

As mentioned in the Introduction, the segmental relaxation of each component may be expected to exhibit dynamic heterogeneity in the lamellar phase. Having noted this, the latter may be moderate at the investigated temperatures. Thus, 


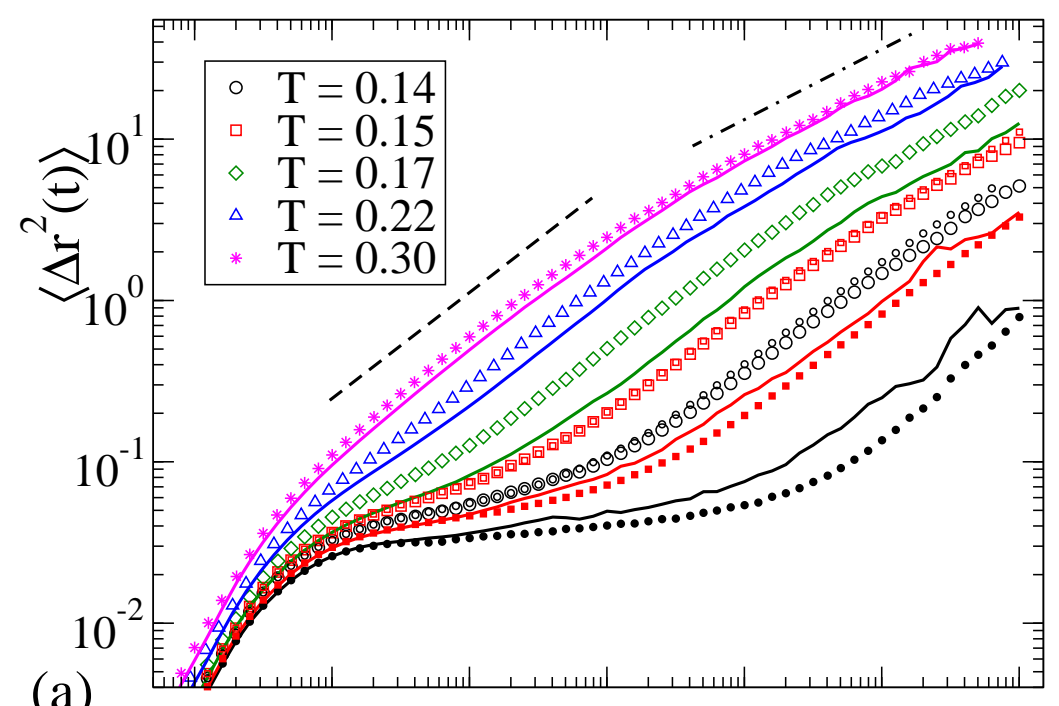

(a)

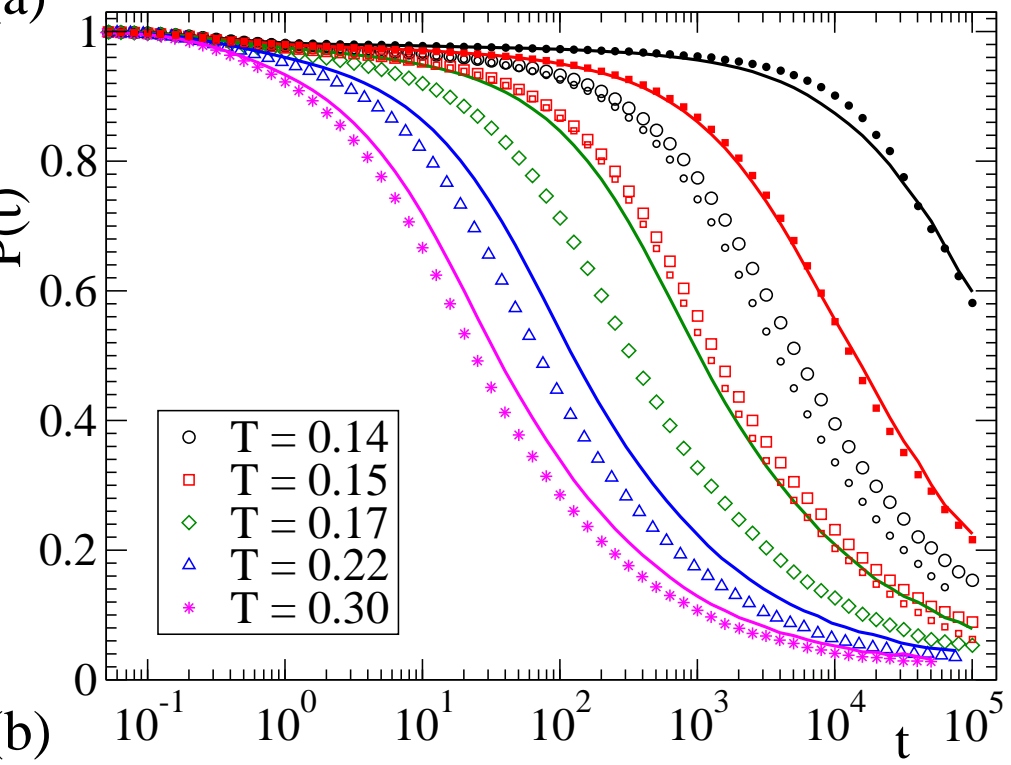

Figure 3.4: Temperature dependence of the mean squared displacement of the $\alpha$ monomers (a) and the orientational correlator of the $\alpha-\alpha$ bonds (b). Large empty symbols and solid lines correspond, respectively, to $\alpha=\mathrm{F}$ and $\mathrm{S}$ in the diblock system. Temperatures for solid lines are, from top to bottom, the same as for large empty symbols (see legend). We also include, as small circles $(T=0.14)$ and small squares $(T=0.15)$ the corresponding data for the homopolymers (empty small symbols: F-homopolymer, filled small symbols: S-homopolymer). Dashed and dashed-dotted lines in panel (a) are power laws $\sim t^{0.65}$ and $\sim t^{0.45}$ respectively. 


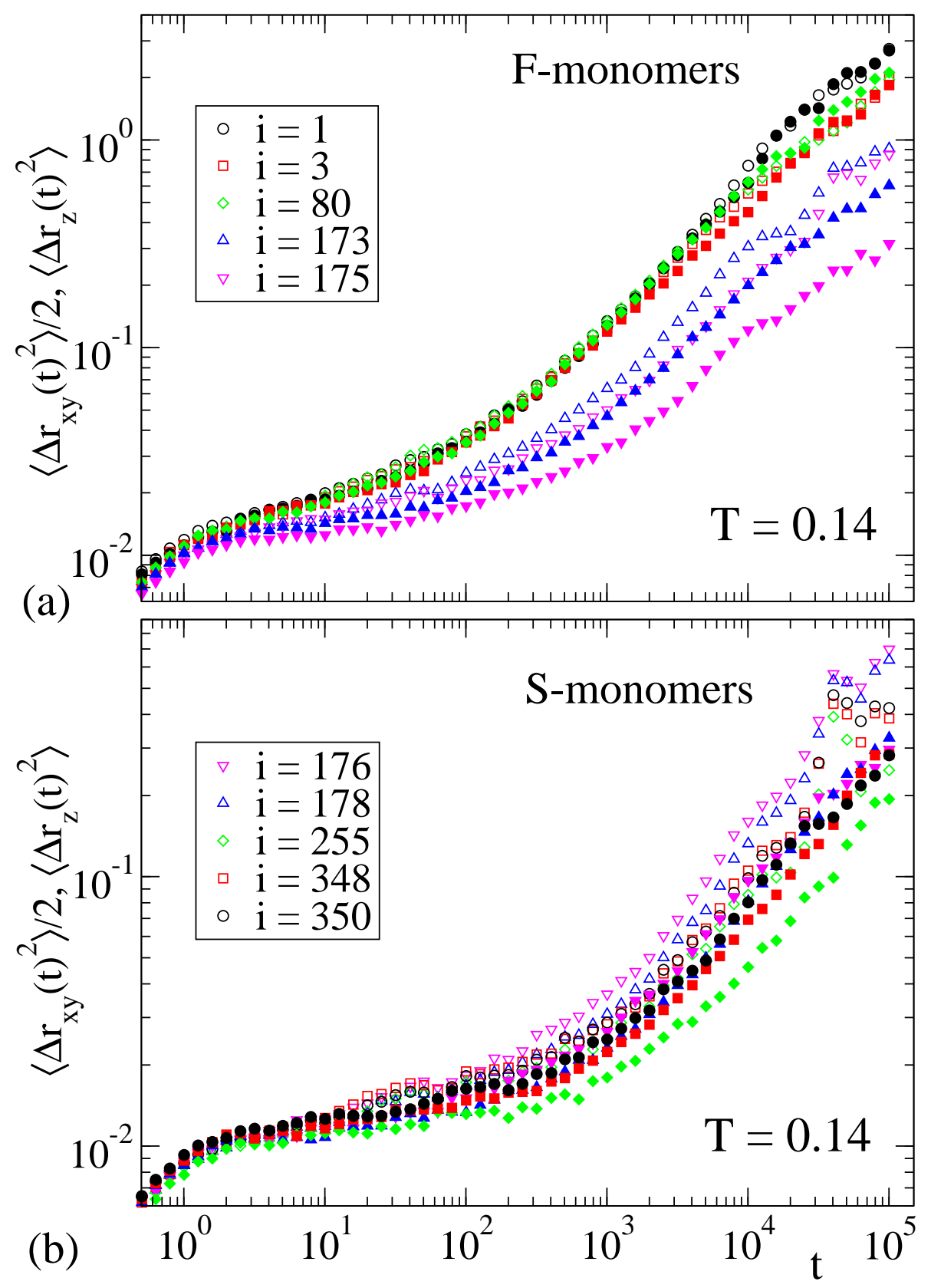

Figure 3.5: For the diblock system at $T=0.14$, components of the mean squared displacement of selected monomers (of index $i$, see legend). Empty and filled symbols (of identical color for a same $i$ ) correspond respectively to $\left\langle\Delta r_{x y}^{2}(t)\right\rangle / 2$ and $\left\langle\Delta r_{z}^{2}(t)\right\rangle$. (a): F-monomers; (b): S-monomers. 


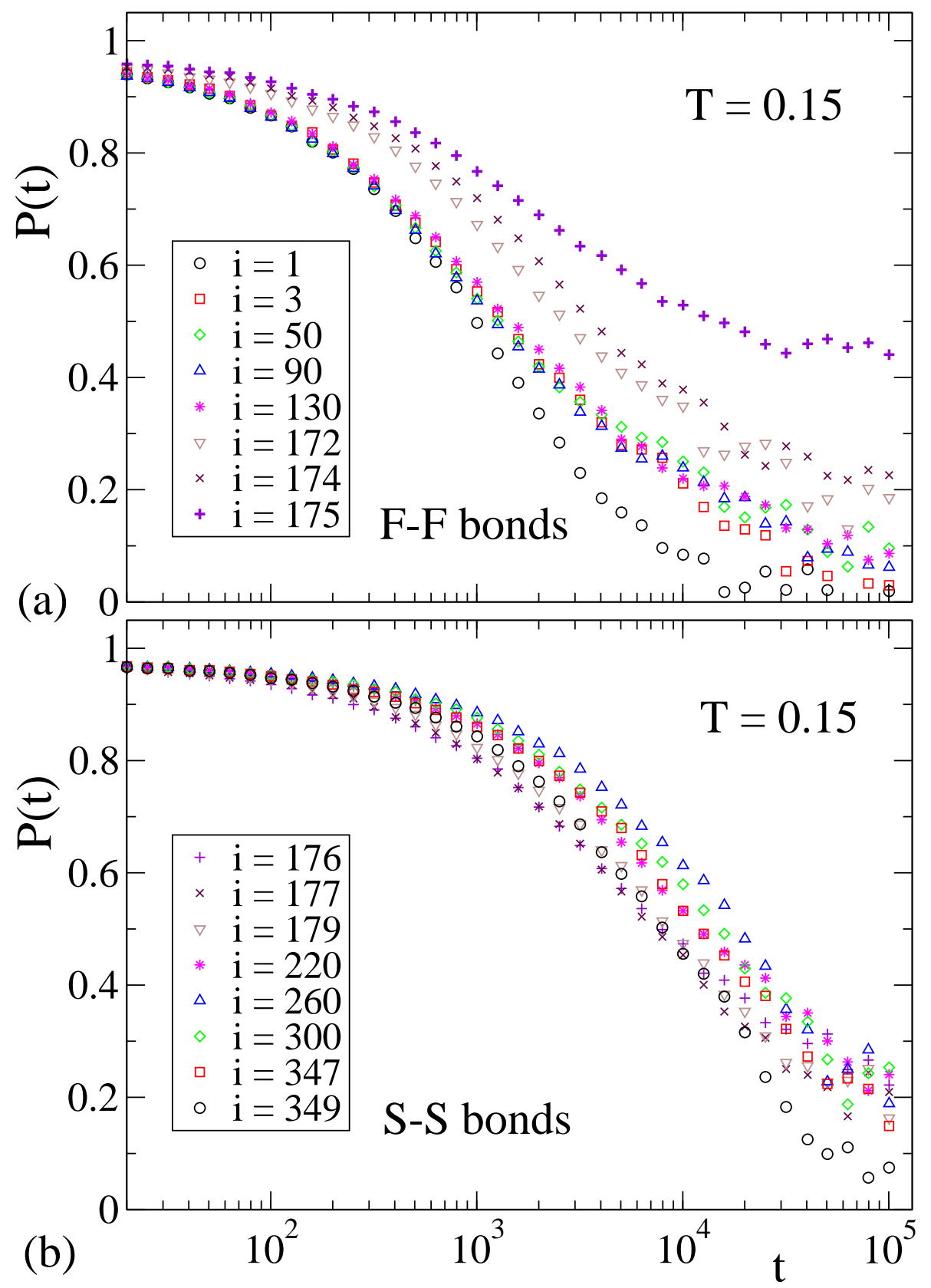

Figure 3.6: For the diblock system at $T=0.15$, orientational correlators for selected monomers (of index $i$, see legend). Results for F-F and S-S bonds are respectively displayed in panels (a) and (b). The panel (a) also includes the data for the F-S bond $(i=175)$. 
the mobility of the junction monomers may be enhanced by the surrounding end monomers, which are intrinsically faster and coexist with the former ones near the interface (see Figure 3.3). This effect may reduce significantly dynamic differences between junction and end monomers. In order to elucidate this point, we show in Figure 3.5, at fixed $T=0.14$, the mean squared displacements of some selected monomers. These results provide a comparison between the dynamics of end monomers $(i=1,3,348$ and 350$)$ and junction monomers $(i=173,175,176$ and 178). We also include results for $i=80$ and 255, corresponding to monomers located at the center of the blocks (in the following denoted as central monomers). Moreover, in order to characterize the anisotropy of the monomer motions, we show the components of the displacements parallel, $\left\langle\Delta r_{x y}^{2}(t)\right\rangle=\left\langle\Delta r_{x}^{2}(t)+\Delta r_{y}^{2}(t)\right\rangle$, and perpendicular, $\left\langle\Delta r_{z}^{2}(t)\right\rangle$, to the lamellar planes. For a correct comparison between both components, data for the parallel component have been rescaled by $1 / 2$. We have selected a reduced set of $i$-values for clarity of presentation of Figure 3.5. Having noted this, the former are representative and cover all the spread observed in the distribution of relaxation times. In particular, the different sets of data in the range $10 \lesssim i \lesssim 160$ for the F-monomers and $210 \lesssim i \lesssim 320$ for the S-monomers are hardly distinguishable from, respectively, the sets $i=80$ and 255 represented in Figure 3.5. In other words, we only observe a significant dynamic spread for the interfacial monomers, i.e., for the junction and end monomers.

Dynamic heterogeneity as a function of the location along the chain exhibits different trends for the F- and S-components. For the F-blocks, the junction monomers are slower than the central and end monomers (Figure 3.5a). This effect is especially visible for the F-monomer directly linked to the S-block $(i=175)$, and in particular for the perpendicular component of the motion, which is strongly slowed down in comparison with the parallel one. No significant anisotropy of the motions is instead observed for the central and end F-monomers, at least within the length scale for the displacements explored in the simulation time window. This is consistent with the experimental observation of a strong reduction of the anisotropy in the diffusivity for lamellae of long entangled chains [6, 30, 31, 40]. 
The former dynamic features for the junction F-monomers can be attributed to their coupling to the dynamics of the neighboring S-monomers, which exhibit an intrinsically slower relaxation. Translational motions of the S-monomers show different trends (Figure 3.5b). In this case the central S-monomers exhibit slower dynamics than the end and junction S-monomers which, according to the distribution $p(i)$ of Figure 3.3, are close (and then dynamically coupled) to faster monomers of the F-domain.

In analogy with the former discussion, Figure 3.6 shows results for the orientational correlators $P(t)$ of selected bonds, at fixed $T=0.15$. The bond $i$ is defined as that connecting the monomers $i$ and $i+1$. Thus, $i=175$ denotes the bond connecting the F- and S-blocks. Reorientation of the junction, end and central bonds follow similar trends to those exhibited by the monomer translations of both F- and S-species. Not surprisingly, the F-S bond connecting the two blocks exhibits a much slower relaxation than the rest of the bonds. Thus, the reorientation of the F-S bond is strongly hindered by the large activation energy produced by the contact between the different species.

The results of Figures 3.5 and 3.6 reveal, for the investigated $T$-range, a moderate heterogeneity in the segmental dynamics as a function of the location along the chain. Similar results are obtained as a function of the distance to the nearest interface. We introduce the layer-dependent mean squared displacement $\left\langle\Delta r^{2}\left(z_{\min }<\Delta z<\right.\right.$ $\left.\left.z_{\max }, t\right)\right\rangle$, defined as the average over the $\alpha$-monomers $(\alpha \in\{\mathrm{F}, \mathrm{S}\})$ which are initially (i.e., at $t=0$ ) located in a layer at a $z$-distance $z_{\min }<\Delta z<z_{\max }$ from the nearest interface. Positive or negative values of $\Delta z$ correspond to monomers initially located in domains of, respectively, their same or the other species. Likewise we introduce the orientational correlator $P\left(z_{\min }<\Delta z<z_{\max }, t\right)$ for $\alpha-\alpha$ bonds. We consider that a bond is initially located at a given layer if its center-of-mass fulfills at $t=0$ the condition $z_{\min }<\Delta z<z_{\max }$. By introducing the former observables for layers perpendicular to the lamellar planes, we characterize the dynamic heterogeneity as a function of the distance to the nearest interface.

Figure 3.7 shows results of $\left\langle\Delta r^{2}(\Delta z, t)\right\rangle$ for the F-monomers [panel (a)] and of 

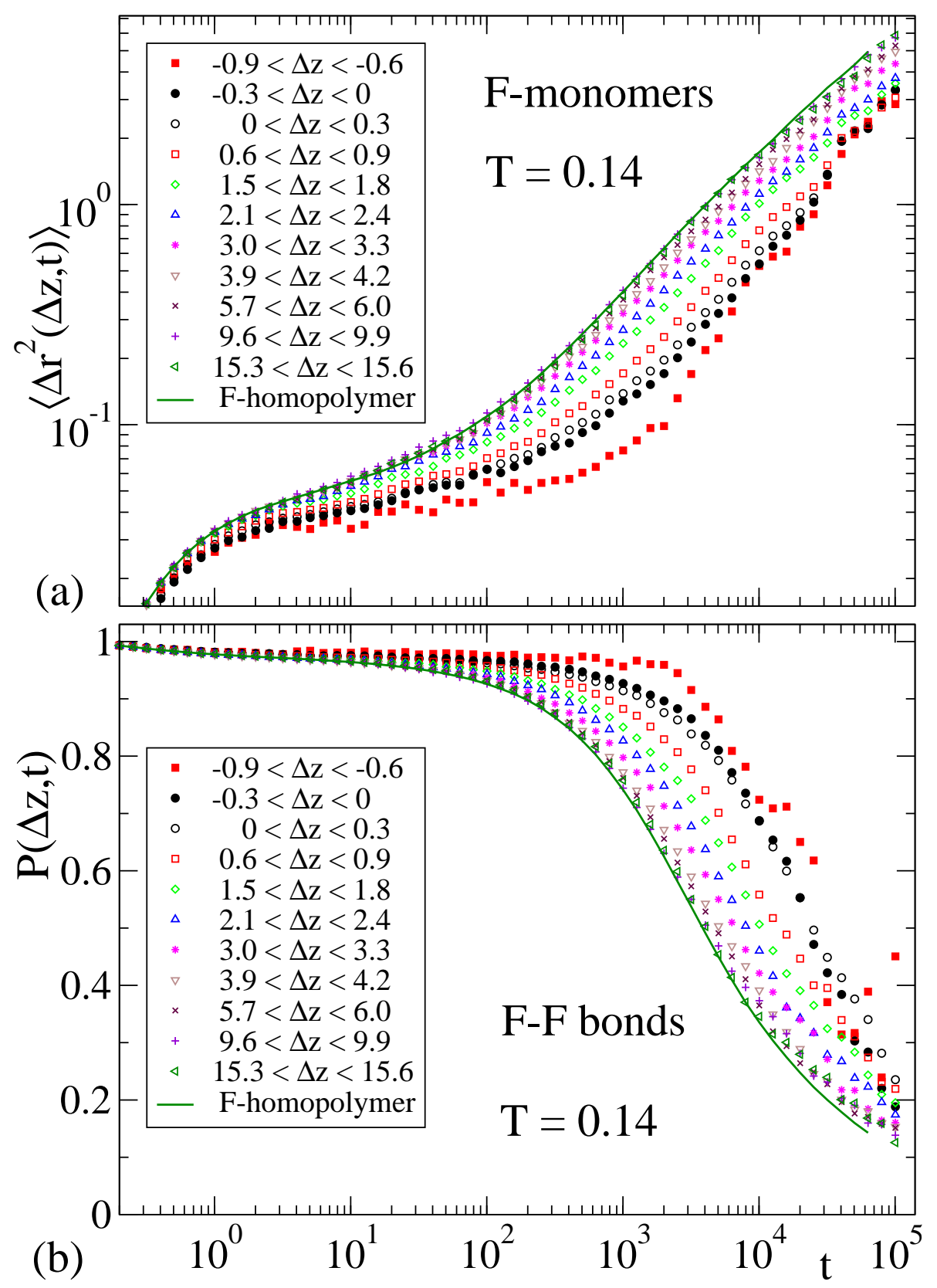

Figure 3.7: Panel (a): symbols are mean squared displacements of F-monomers for different layers (see text). Positive and negative values of $\Delta z$ correspond to monomers located, at $t=0$, respectively in the F- and S-domains. All data correspond to $T=0.14$. Panel (b): as panel (a) for the layer-dependent orientational correlators $\mathrm{P}(\Delta z, \mathrm{t})$ of the F-F bonds (symbols). Lines in both panels are the data for the F-homopolymer. 

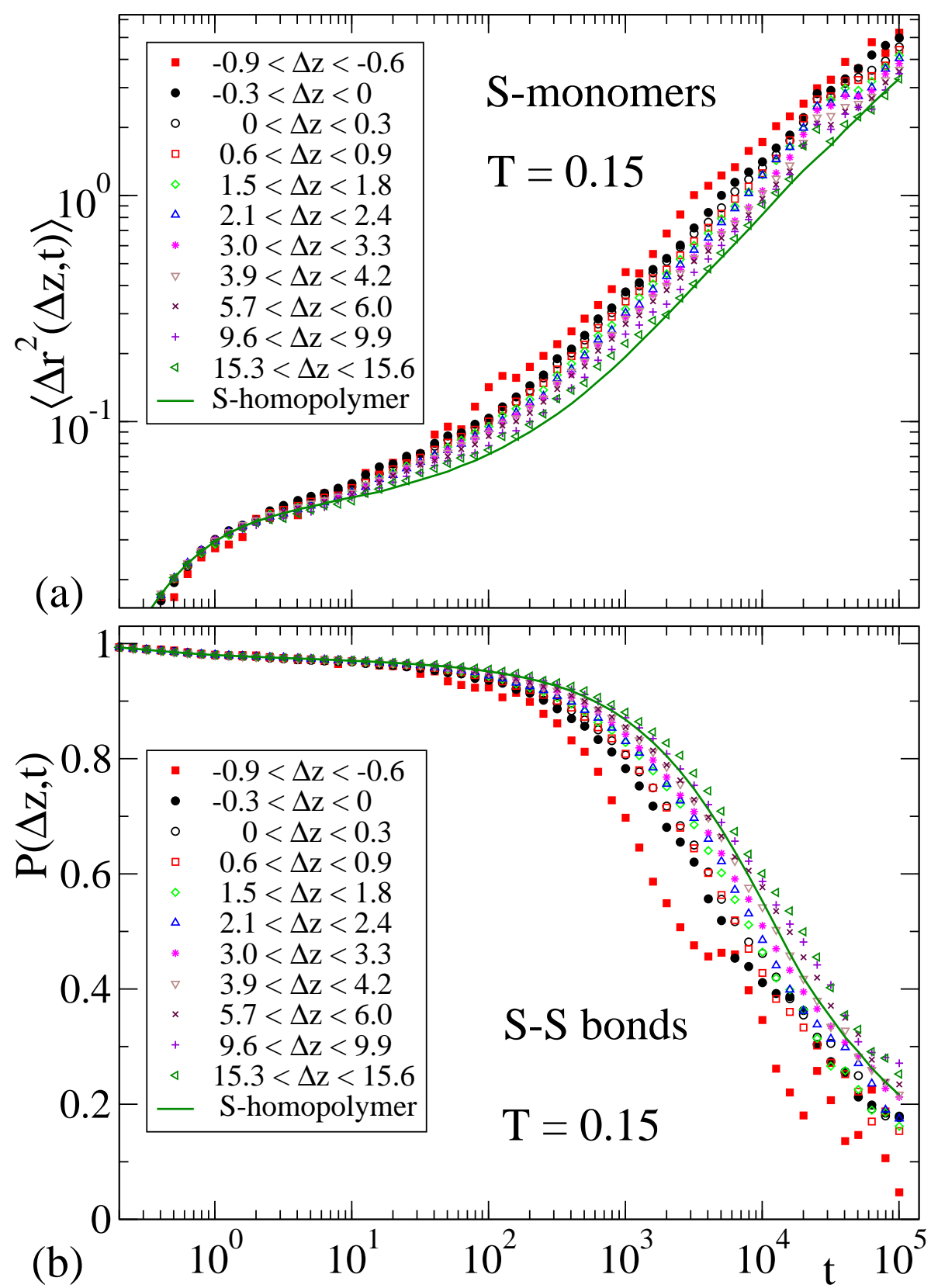

Figure 3.8: As Figure 3.7 for S-monomers and S-S bonds. All data correspond to $T=0.15$. 
$P(\Delta z, t)$ for the F-F bonds [panel (b)]. A moderate dispersion, over about one decade, is again observed in the respective relaxation times. Not surprisingly, the relaxation of the F-monomers and F-F bonds initially located at layers within the slow S-domain is slower than the relaxation of those located at the center of the F-domains, with a monotonic crossover between both limits. The dynamics of the F-homopolymer is recovered as the center of the F-domain is approached. Thus, for distances $\Delta z \gtrsim 4 \sigma$ from the nearest interface the data for the F-blocks are almost indistinguishable from those of the F-homopolymer. Figure 3.8 shows the corresponding results of $\left\langle\Delta r^{2}(\Delta z, t)\right\rangle$ and $P(\Delta z, t)$ for the S-monomers and S-S bonds. In this case the structural relaxation is slowed down and approaches that of the S-homopolymer on moving from the interface to the center of the S-domain. 


\section{Chapter 4}

\section{Discussion}

As for the global bond correlators $P(t)$, we define the relaxation times $\tau$ for the layer-dependent correlators as the times at which $P(\Delta z, \tau)=e^{-1}$. We have fitted the so-obtained relaxation times for the F-F bonds to an empirical Vogel-FulcherTamann (VFT) law, $\tau=\tau_{\infty} \exp \left[E / k_{\mathrm{B}}\left(T-T_{0}\right)\right]$. The latter is usually invoked in the analysis of the structural relaxation in glass-forming systems [47, 48]. Figure 4.1 shows some representative fits for F-F bonds close to the interface and at the center of the F-domains. The fit parameters $\tau_{\infty}, E$, and $T_{0}$ are displayed in Figure 4.2 as a function of the layer position. For comparison we include the results for the global correlator $P(t)$ (i.e, averaged over all the F-F bonds), both for the F-blocks in the lamellar phase and for the F-homopolymer.

The values of the VFT parameters for $P(\Delta z, t)$, obtained from the fully free fits, vary with the distance to the interface. The VFT parameters are strongly coupled and descriptions with the same quality of Figure 4.1 can be achieved by means of slight variations of those obtained from the fully free fits (see error bars in Figure 4.2). This is the case by leaving $T_{0}$ as the only free parameter and fixing the values of the attemp period $\tau_{\infty}$ and the VFT activation energy $E$. Namely we fix $\tau_{\infty}=12.4$, i.e., the value obtained for the global $P(t)$ of both the F-homopolymer and the F-blocks in the lamellar phase (Figure 4.2a). For the VFT activation energy we select two fixed values $E=0.300$ and $E=0.306$. These very similar values are those obtained for the global $P(t)$ of respectively the F-homopolymer and the F- 


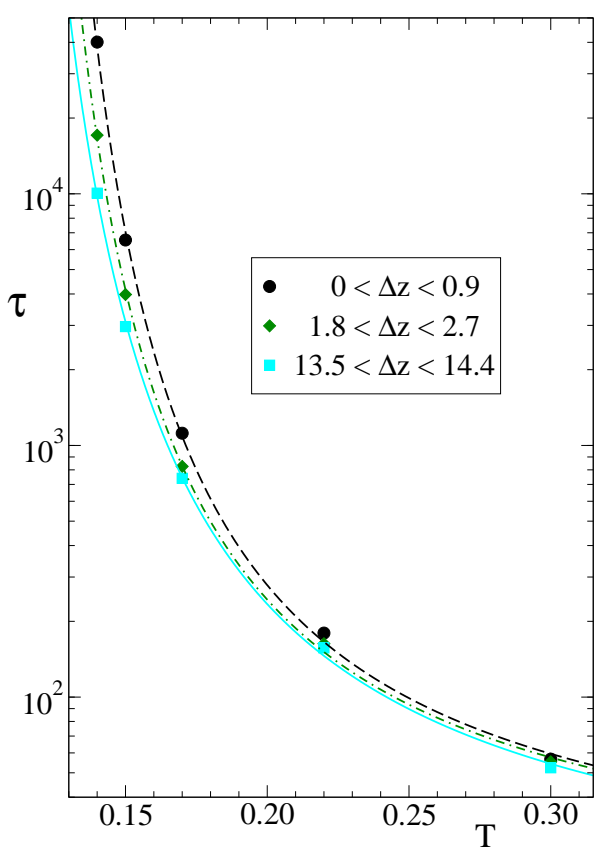

Figure 4.1: Symbols: relaxation times of the correlators $P(\Delta z, t)$ for the F-F bonds in three selected layers. Lines: Fits to an VFT law $\tau=\tau_{\infty} \exp \left[E / k_{\mathrm{B}}\left(T-T_{0}\right)\right]$.

blocks in the lamellar phase (Figure 4.2b). Not surprisingly, this fitting scheme with fixed $\tau_{\infty}$ and $E$ yields a smoother layer-dependence of $T_{0}$ than in the fullyfree fit (see Figure 4.2c). The so-obtained $T_{0}(\Delta z)$ decreases by moving from the interface to the center of the F-domain. Initially it shows a sharp decay, until a distance from the interface of about $4 \sigma$, for which the $T_{0}$ of the F-homopolymer is already approached. This is consistent with the relaxation of $P(\Delta z, t)$ observed in Figure 3.7b, which is similar to that of the F-homopolymer for $\Delta z \gtrsim 4 \sigma$, while it is slowed down on approaching the interface (as expected from an increasing $T_{0}$ ).

Figure 4.3 shows the corresponding VFT parameters for the correlators of the S-S bonds. The parameters obtained from fully free fits show opposite trends to those of Figure 4.2 for the F-F bonds. It is noteworthy that the interfacial effects for $T_{0}(\Delta z)$ seem to emerge again in the range $\Delta z \lesssim 4 \sigma$. A forced fit by fixing the values of $\tau_{\infty}$ and $E$ to those of the S-homopolymer seems less justified than in the case of the F-F bonds. Indeed the so-obtained values of $T_{0}(\Delta z)$ exhibit, near the interface, a much weaker variation than those obtained from fully free fits. Having said this, it must 


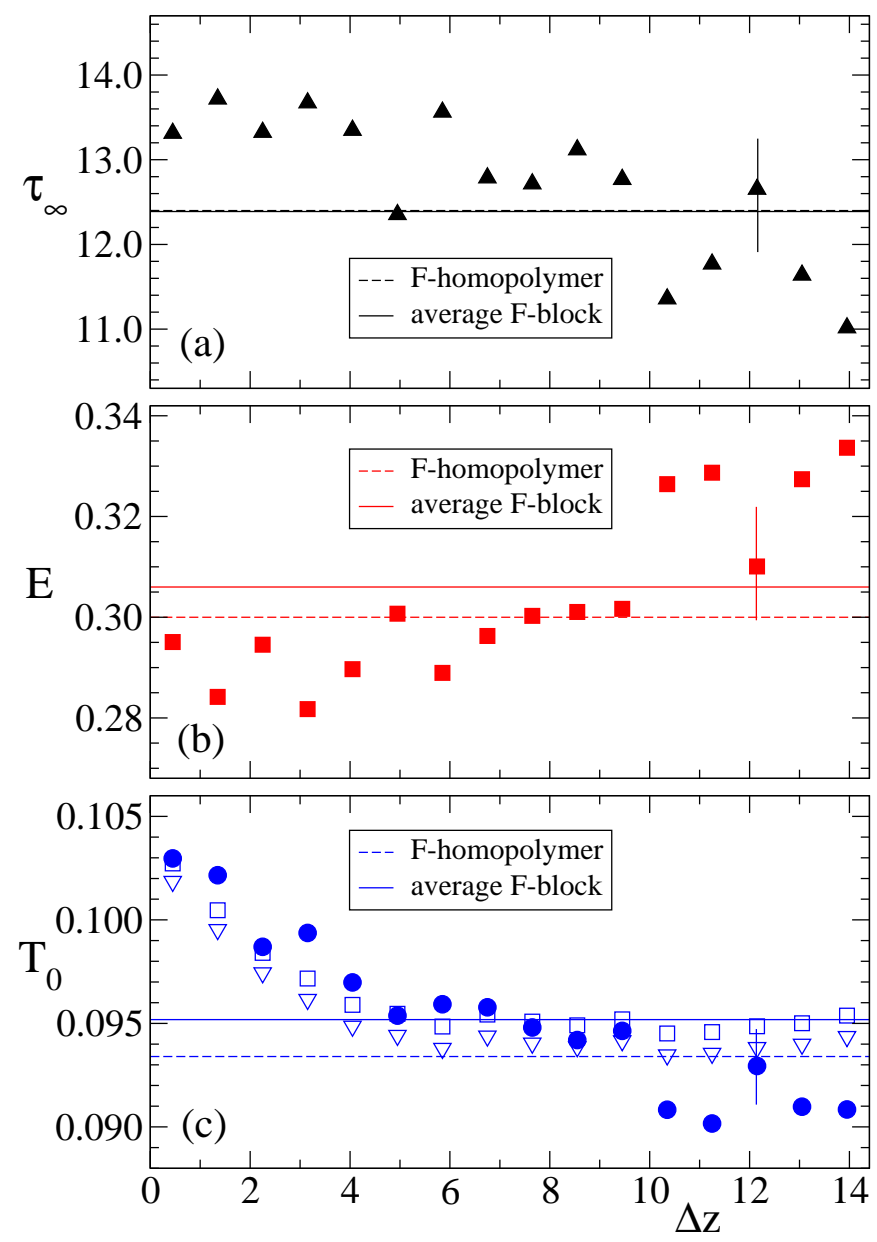

Figure 4.2: Horizontal lines: VFT parameters for the relaxation times of the global correlators $P(t)$ for the F-blocks (solid lines) and the F-homopolymer (dashed lines). Symbols: VFT parameters for the relaxation times of the layer-dependent correlators $P(\Delta z, t)$ for the F-F bonds. Data in panels (a), (b) and (c) correspond respectively to $\tau_{\infty}, E$, and $T_{0}$. Filled symbols are data obtained from fully free fits. Vertical lines are typical error bars. Empty symbols in panel (c) are data obtained from VFT fits with free $T_{0}$ and fixed values of $\tau_{\infty}$ and $E$. Namely we fix $\tau_{\infty}=12.4$, i.e, the value obtained for the global $P(t)$ of both the F-homopolymer and the F-blocks [see panel (a)]. For the VFT activation energy we fix $E=0.300$ (squares) and $E=0.306$ (triangles), which are respectively the values obtained for the global $P(t)$ in the F-homopolymer and in the F-blocks [see horizontal lines in panel (b)]. 


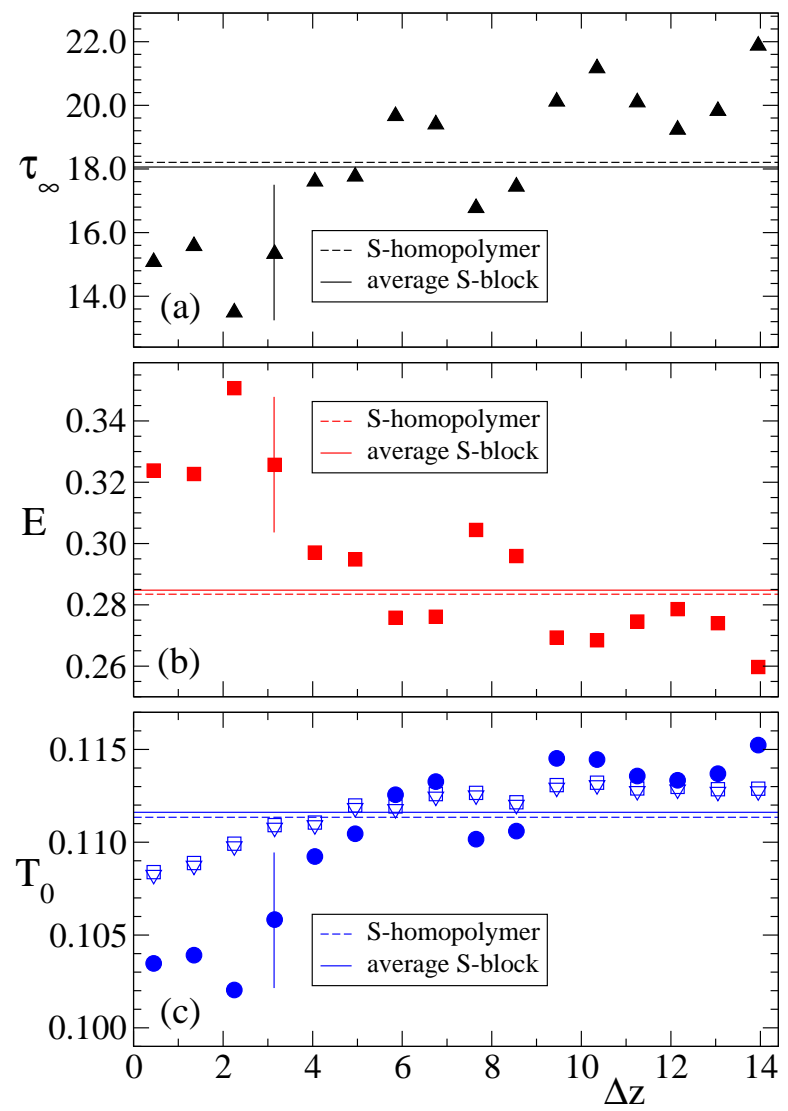

Figure 4.3: As Figure 4.2 for the S-component.

be noted that unlike for the F-F bonds, at $T=0.14$ the orientational correlators for the S-S bonds do not decay to $e^{-1}$ within the simulation window, and therefore the corresponding relaxation times $\tau$ at $T=0.14$ are not available for the corresponding VFT fits. For this reason the VFT parameters from fully free fits in Figure 4.3 have larger uncertainties than those for the F-F bonds in Figure 4.2, and the discrepancies between the different sets of $T_{0}(\Delta z)$ in Figure $4.3 \mathrm{c}$ must be taken with care.

In the following we discuss the results obtained for the fast F-component in comparison with experimental observations. In particular, we construct layer-dependent VFT curves according to the analysis performed by Lorthioir et al. in Ref. [43], for BDS data of the structural relaxation of poly(dimethylsiloxane) (PDMS) in the diblock lamellar phase with poly(styrene) (PS). It must be noted that PDMS $\left(T_{\mathrm{g}} \approx 145 \mathrm{~K}\right)$ has a lower glass transition temperature than PS $\left(T_{\mathrm{g}} \approx 370 \mathrm{~K}\right)$, and 
therefore the F-homopolymer in the present model qualitatively plays the role of the PDMS. By taking the values of $T_{0}(\Delta z)$ for fixed $\tau_{\infty}=12.4$ and $E=0.300$ (empty squares in Figure 4.2c), we construct for each layer the corresponding VFT curve extrapolated to low temperatures, below the range investigated by simulations. Indeed the analysis of Ref. [43] is equivalent to assume, as in our case, values of $\tau_{\infty}$ and $E$ constant and identical to those of the corresponding homopolymer, and leaving $T_{0}(\Delta z)$ as the only layer-dependent parameter.

The extrapolated VFT curves for the reorientation of the F-F bonds are shown in Figure 4.4. The set of curves (not shown) constructed with the VFT parameters obtained from fully-free fits (filled symbols in Figure 4.2) exhibits the same qualitative trends. The relaxation times for the different layers progressively spread as temperature decreases. Thus, by assuming the validity of the extrapolation, we observe at $T=0.113$ a variation in the relaxation times of about 5 decades from the interface to the center of the F-domains. At the former temperature the relaxation time in the inmediate neighborhood of the interface is $10^{14}$, which roughly corresponds to $10^{2}-10^{3}$ seconds in real systems (see Chapter 2), i.e., the usual time scale for defining the laboratory glass transition temperature [47, 48]. Consistently with the observations in Figures 3.7b and 4.2c, the observed spread in the relaxation times esentially emerges at distances from the interface smaller than about $4 \sigma$. Note that the small differences in the extrapolated VFT curves at large values of $\Delta z$ originate from the statistical error in the used values of $T_{0}(\Delta z)$ (see Figure 4.2c).

The results of Figures 3.7, 4.2 and 4.4 are consistent with the physical interpretation of the experiments of Ref. [43], which proposes the existence of gradients of mobility originating from gradients of VFT temperatures, $T_{0}(\Delta z)$. The dynamic range probed by the global response of the PDMS-segments in Ref. [43] extends over frequencies lower than $10^{5} \mathrm{~Hz}$. By using the conversion factors from simulation to real units (see Chapter 2), the former dynamic range qualitatively correspons to temperatures for which the relaxation time of the global $P(t)$ is $\tau \gtrsim 10^{7}$. At such temperatures the layer-dependent times of Figure 4.4 spread over at least three decades. This is qualitatively consistent with the distributions of times $\tau(\Delta z)$ ob- 


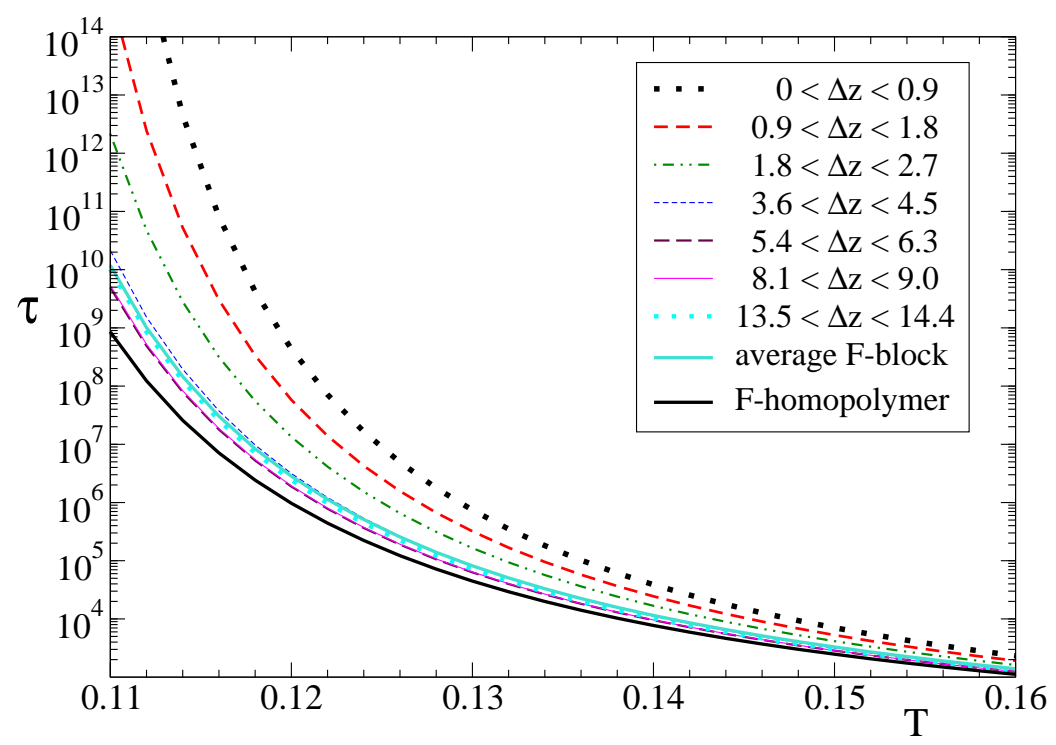

Figure 4.4: VFT curves for the reorientation of the F-F bonds, extrapolated to low temperatures (see text for details).

tained from the experimental analysis of Ref. [43]. There it is also proposed that the gradients of mobility extend from the interface to some distance $d$, beyond which the PDMS-segments in the lamellar domains esentially follow the same dynamic behavior as in the PDMS-homopolymer. The estimated spatial extension of the gradients is $d \sim 4 \mathrm{~nm}$ [43]. This value is qualitatively consistent with the distance, $\Delta z \approx 4 \sigma$, at which the VFT temperature $T_{0}(\Delta z)$ and layer-dependent dynamic observables approach those of the F-homopolymer in our simulations (see Figures 3.7, 4.2 and 4.4).

According to the interpretation of Ref. [43] the gradients of mobility, which are directly related with the obtained gradients of VFT temperatures $T_{0}(\Delta z)$, originate from gradients of density. Inspection of Figures 3.2 and 4.2 suggests that the latter is esentially an interfacial effect. Thus, $\rho(\Delta z)$ and $T_{0}(\Delta z)$ become constant (layerindependent), and equal within error bars to the values for the F-homopolymer, at similar distances from the interface. These distances are $\Delta z \gtrsim 3 \sigma$ for $\rho(\Delta z)$ (Figure 3.2) and $\Delta z \gtrsim 4 \sigma$ for $T_{0}(\Delta z)$ (Figure 4.2). 


\section{Chapter 5}

\section{Conclusions}

We have performed simulations of a simple bead-spring model for strongly segregated lamellar phases of diblock copolymers, with blocks of different mobility. We have characterized the heterogeneity of the segmental dynamics associated to the glass transition. This has been done by computing monomer translations and bond reorientations, as a function of both the location along the chain and the distance to the nearest interface. This analysis reveals moderate gradients of mobility in the investigated temperature range, which qualitatively probes time scales of up to hundreds nanoseconds. Thus, the obtained distribution of relaxation times spreads over about one decade. This result is relevant for the interpretation of experiments probing the former dynamic window, e.g. by means of neutron spin-echo techniques [84], suggesting that strong gradients of mobility and broad distributions of relaxation times should not be invoked in the data analysis.

However, the extrapolation of the simulation results to lower temperatures (i.e., to much longer time scales) suggests an increasing dispersion over several time decades. The extrapolated results are consistent with the interpretation of BDS experiments, which relate gradients of mobility with gradients of the VFT temperature. The simulations reveal, for the fast block, a decrease of the VFT temperature on moving from the interface to the center of the lamellar domain. The opposite effect is observed for the slow block. The structural relaxation and VFT temperature within the lamellar domains approach those of the respective homopolymers at a 
distance from the interface of about 2-4 nm, in agreement with proposed values from BDS experiments. This distance roughly corresponds to the interfacial thickness. Therefore, gradients of mobility emerge esentially as an interfacial effect. They are not related with long-range gradients of density along the lamellar domains. The latter are indeed absent, and the density of the corresponding homopolymers is only perturbed in the interfacial region. 


\section{Bibliography}

[1] Bates, F.S, Fredrickson, G.H. Annu. Rev. Phys. Chem. 1990, 41, 525.

[2] F. S. Bates. Science. 1991, 251, 898-905.

[3] Fredrickson, G.H, Bates, F.S. Annu. Rev. Mat. Sci. 1996, 26, 501.

[4] Alexandridis, P, Lindman B. (eds.) Amphiphilic Block Copolymers; Elsevier: Amsterdam, Netherlands, 2000.

[5] Hamersky, M.W, Hillmyer, M.A, Tirrell, M, Bates, F.S, Lodge, T.P, von Meerwall, E.D. Macromolecules 1998, 31, 5363.

[6] Lodge, T.P, Dalvi, M.C. Phys. Rev. Lett. 1995, 75, 657.

[7] R. W. Kelsall1, I.W. Hamley, M.Geoghegan, Self-Assembling Nanostructured Molecular Materials and Devices, Wiley, 2005.

[8] M.Sczwarc, Living Polymers and Mechanisms of Anionic Polymerization, (Advances in Polymer Science); Springer, 1983.

[9] W. Matsen, F. S. Bates, Macromolecules 1996, 29, 2443.

[10] Shull, K. R. Macromolecules 1992 , 25, 2122.

[11] Shull, K. R, Mayes, A. M, Russell, T. P. Macromolecules, 1993, 26, 3929.

[12] Matsen, M. W, Bates. F. S. Macromolecules 1996, 29, 1091.

[13] Matsen, M. W, Bates. F. S. J. Chem. Phys 1997, 106, 2436. 
[14] Ko.M.B, Mattice.W.L. Macromolecules 1995, 28, 6871.

[15] Cavicchi.K.A, Lodge.T.P. Macromolecules 2004, 37, 6004.

[16] Khanna, V, Kim, B.J, Hexemer, A, Mates, T.E, Kramer, E.J, Li, X, Wang, J, Hahn, S.F. Macromolecules 2007, 40, 2443.

[17] Willner,L. Lund,R, Monkenbusch.M, Holderer.O, Colmenero.J, Richter.D. Soft Matter 2010, 6, 1559.

[18] Rittig, F, Kärger, J, Papadakis, C.M, Fleischer, G, Almdal, K, Štěpánek, P. Macromolecules 2001, 34, 868.

[19] Hoffmann, A, Sommer, J.U, Blumen, A. J. Chem. Phys. 1997, $107,7559$.

[20] Yokoyama, H, Kramer, E.J. Macromolecules 1998, 31, 7871.

[21] Fleischer, G, Rittig, F, Kärger, J, Papadakis, C.M, Mortensen, K, Almdal, K, Štěpánek, P. J. Chem. Phys. 1999, 111, 2789.

[22] Yokoyama, H, Kramer, E.J, Fredrickson, G.H. Macromolecules 2000, 33, 2249.

[23] Cavicchi, K.A, Lodge, T.P. Macromolecules 2003, 36, 7158.

[24] Papadakis, C.M, Rittig, F, Almdal, K, Mortensen, K, Štěpánek, P. Eur. Phys. J. E 2004, 15, 359 .

[25] Moreno, A.J, Colmenero, J. Macromolecules 2009, 42, 8543.

[26] Lund, R, Willner, L, Alegría, A, Colmenero, J, Richter, D. Macromolecules 2008, 41, 511 .

[27] Stühn, B, Stickel, F. Macromolecules 1992, 25, 5306.

[28] Hoffmann, A, Koch, T, Stühn, B. Macromolecules 1993, 26, 7288.

[29] Ehlich, D, Takenaka, M, Hashimoto, T. Macromolecules 1993, 26, 492.

[30] Dalvi, M.C, Eastman, C.E, Lodge, T.P. Phys. Rev. Lett. 1993, 71, 2591. 
[31] Dalvi, M.C, Lodge, T.P. Macromolecules 1993, 26, 859.

[32] Haliloglu, T, Balaji, R, Mattice, W.L. Macromolecules 1994, 27, 1473.

[33] Karatasos, K, Anastasiadis, S.H, Floudas, G, Fytas, G, Pispas, S, Hadjichristidis, N, Pakula, T. Macromolecules 1996, 29, 1326.

[34] Hoffmann, A, Sommer, J.U, Blumen, A. J. Chem. Phys. 1997, 106, 6709.

[35] Alig, I, Floudas, G, Avgeropoulos, A, Hadjichristidis, N. Macromolecules 1997, 30, 5004 .

[36] Pakula, T, Karatasos, K, Anastasiadis, S.H, Fytas, G. Macromolecules 1997, $30,8463$.

[37] Hamersky, M.W, Tirrell, M, Lodge, T.P. Langmuir 1998, 14, 6974.

[38] Murat, M, Grest G.S, Kremer, K. Europhys. Lett. 1998, 42, 401.

[39] Murat, M, Grest G.S, Kremer, K. Macromolecules 1999, 32, 595.

[40] Anastasiadis, S.H, Rittig, F, Chrissopoulou, K, Fleischer, G, Fytas, G, Semenov, A.N, Kärger, J, Xenidou, M, Pispas, S, Hadjichristidis, N. Europhys. Lett. 2000, 51, 68 .

[41] Kyritsis, A, Pissis, P, Mai, S.-M, Booth, C. Macromolecules 2000, 33, 4581.

[42] Dollase, T, Graf, R, Heuer, A, Spiess, H. W. Macromolecules 2001, 34, 298.

[43] Lorthioir, C, Alegría, A, Colmenero, J, Deloche, B. Macromolecules 2004, 37, 7808.

[44] Encinar, M, Guzmán, E, Prolongo, M. G, Rubio, R. G, Sandoval, C, GonzálezNilo, F, Gargallo, L, Radić, D. Polymer 2008, 49, 5650.

[45] Mok, M. M, Masser, K. A, Runt, J, Torkelson, J. M. Macromolecules 2010, 43, 5740 . 
[46] del Valle-Carrandi, L, Alegría, A, Colmenero, J. Eur. Phys. J. Special Topics 2010, 189, 257.

[47] Debenedetti, P.G. Metastable Liquids; Princeton University Press: Princeton, 1996.

[48] Binder, K, Kob, W. Glassy Materials and Disordered Solids; World Scientific: Singapore, 2005.

[49] Lai, P.-Y, Binder, K. J. Chem. Phys. 1992, 97, 586.

[50] Neelov, I.M, Binder, K. Macromol. Theory Simul. 1995, 4, 1063.

[51] Baschnagel, J, Varnik, F. J. Phys.: Condens. Matter 2005, 17, R851.

[52] Varnik, F, Baschnagel, J, Binder, K. Phys. Rev. E 2002, 65, 021507.

[53] Torres, J.A, Nealey, P.F, de Pablo, J.J. Phys. Rev. Lett. 2000, 85, 3221.

[54] Morita, H, Tanaka, K, Kajiyama, T, Nishi, T, Doi, M. Macromolecules 2006, $39,6233$.

[55] Peter, S, Napolitano, S, Meyer, H, Wübbenhorst, M, Baschnagel, J. Macromolecules 2008, 41, 7729 .

[56] Peter, S, Meyer, H, Baschnagel, J. J. Polym. Sci., Part B: Polym. Phys. 2006, 44, 2951.

[57] Scheidler, P, Kob, W, Binder, K. Europhys. Lett. 2000, 52, 277.

[58] Scheidler, P, Kob, W, Binder, K. J. Phys. Chem. B 2004, 108, 6673.

[59] Eral, H.B, van den Ende, D, Mugele, F, Duits, M.H.G. Phys. Rev. E 2009, 80, 061403.

[60] Metropolis. N, A.Rosenbluth, M. Rosenbluth, A. Teller, E. Teller, J. Chem. Phys, 1953, 21, 1087-1092.

[61] Alder, B.J; Wainwright.T.E. J. Chem. Phys. 1957, 27, 1208. 
[62] M.P Allen and D. J. Tildesley, Computer Simulation of Liquids, $2^{\text {nd }}$ Ed Plenum, New York, 1996.

[63] D. Frenkel and B. Smit, Understanding Molecular Simulation; Academic Press (Elsevier): San Diego, 1996.

[64] D.C Rapaport, The Art of Molecular Dynamics Simulation, $2^{\text {nd }}$ Ed Cambridge, 2004.

[65] Paul, W.; Yoon, D.Y.; Smith, G.D. J. Chem. Phys. 1995, 103, 1702.

[66] Paul, W.; Smith, G.D.; Yoon, D.Y. Macromolecules 1997, 30, 7772.

[67] Lyulin, A.V; Michels, M.A.J. Macromolecules 2002, 35, 9595.

[68] Rossi, G.; Monticelli, L.; Puisto, S.R.; Vattulainen, I.; Ala-Nissila, T. Soft Matter 2011, 7, 698.

[69] Grest, G.S.; Kremer, K. Phys. Rev. A 1986, 33, R3628.

[70] Kremer, K, Grest, G.S. J. Chem. Phys. 1990, 92, 5057.

[71] Martyna, G.J, Tuckerman, M.E, Tobias, D.J, Klein, M.L. Mol. Phys. 1996, 87, 1117.

[72] Anderson, J.A, Lorenz, C.D, Travesset, A. J. Chem. Phys. 2008, 128, 184906.

[73] Grest, G.S, Lacasse, M.D, Kremer, K, Gupta, A.M. J. Chem. Phys. 1996, 105, 10583.

[74] Loison, C, Mareschal, M, Kremer, K, Schmid, F. J. Chem. Phys. 2003, 119, 13138.

[75] Schultz, A. J, Hall, C. K, Genzer, J. J. Chem. Phys. 2004, 120, 2049.

[76] Almdal, K, Rosedale, J.H, Bates, F.S, Wignall, G.D, Fredrickson, G.H, Phys. Rev. Lett. 1990, 65, 1112.

[77] Jain, T.S, de Pablo, J.J. Phys. Rev. Lett. 2004, 92, 155505. 
[78] Baljon, A.R.C, van Weert M.H.M, de Graaff, R.B, Khare, R. Macromolecules 2005, 38, 2391.

[79] Gulati, H.S, Hall, C.K, Jones, R.L, Spontak, R.J. J. Chem. Phys. 1996, 105, 7712.

[80] Pastorino, C, Binder, K, Kreer, T, Müller, M. J. Chem. Phys. 2006, 124, 064902.

[81] Arbe, A, Colmenero, J, Soft Matter 2007, 3, 1474.

[82] Doi, M, Edwards, S.F. The Theory of Polymer Dynamics; Oxford University Press: Oxford, U.K., 1986.

[83] Everaers, R, Sukumaran, S.K, Grest, G.S, Svaneborg, C, Sivasubramanian, A, Kremer, K. Science 2004, 303, 823.

[84] Richter, D, Monkenbusch, M, Arbe, A, Colmenero, J. Neutron Spin Echo in Polymer Systems. In Advances in Polymer Science; Springer-Verlag: Berlin, 2005; Vol. 174. 